\title{
QUADRATIC SOLUTIONS OF QUADRATIC FORMS
}

\author{
JÁNOS KOLLÁR
}

The aim of this note is to study solutions of a homogeneous quadratic equation $q\left(x_{0}, \ldots, x_{n+1}\right)=0$, defined over a field $k$, where the $x_{i}=h_{i}\left(u_{0}, \ldots, u_{r}\right)$ are themselves homogeneous polynomials of a given degree $d$. Equivalently, we are looking at rational maps defined over $k$,

$$
\Phi: \mathbb{P}^{r} \rightarrow Q^{n}:=\left(q\left(x_{0}, \ldots, x_{n+1}\right)=0\right) \subset \mathbb{P}^{n+1}
$$

from projective $r$-space to an $n$-dimensional quadric hypersurface. We denote the set of such maps by $\operatorname{Map}_{d}\left(\mathbb{P}^{r}, Q^{n}\right)(k)$. It is easy to see that there is a projective algebraic set $\operatorname{Map}_{d}\left(\mathbb{P}^{r}, Q^{n}\right)$, defined over $k$, whose $K$ points can be naturally identified with $\operatorname{Map}_{d}\left(\mathbb{P}^{r}, Q^{n}\right)(K)$ for any field extension $K \supset k$. Thus we aim to describe the algebraic sets $\operatorname{Map}_{d}\left(\mathbb{P}^{r}, Q^{n}\right)$ and give concrete parametrization of their $k$ points.

If $d=1$ then the image of $\Phi$ is a linear subspace of $Q^{n}$, hence the study of linear solutions of quadratic equations is essentially equivalent to the theory of orthogonal Grassmannians. These are homogeneous spaces under the orthogonal group $\operatorname{Aut}\left(Q^{n}\right)=\operatorname{PGO}(q)$ and quite well understood, see Section 8

Another extreme case is when $r=1$. Solving $q\left(x_{0}, \ldots, x_{n+1}\right)=0$, where the $x_{i}=h_{i}(u, v)$ are homogeneous polynomials in 2 variables $u, v$ is equivalent to studying maps $\mathbb{P}^{1} \rightarrow Q^{n}$. In algebraic geometry there has been considerable interest in understanding maps from $\mathbb{P}^{1}$ to a given variety. Most of these works deal with more general varieties over algebraically closed fields. Quadrics are special cases of homogeneous spaces KP01 and of low degree hypersurfaces HRS04. See the introductory notes AK03, Kol10 or the more complete treatment given in Kol96. for details. The existence of maps $\mathbb{P}^{1} \rightarrow X$ over finite fields is studied in [Kol08, but not much is known about the structure of these spaces over arbitrary fields. For quadrics, we prove the following in Section 2 ,

Theorem 1. Let $Q^{n}$ be a smooth quadric of dimension $n \geq 3$. Then

$$
\operatorname{Map}_{d}\left(\mathbb{P}^{1}, Q^{n}\right) \stackrel{b i r}{\sim}\left\{\begin{array}{l}
Q^{n} \times \mathbb{P}^{n d} \text { if } d \text { is even, and } \\
\operatorname{OG}\left(\mathbb{P}^{1}, Q^{n}\right) \times \mathbb{P}^{n d-n+3} \quad \text { if } d \text { is odd }
\end{array}\right.
$$

where $\stackrel{\text { bir }}{\sim}$ denotes birational equivalence and $\mathrm{OG}\left(\mathbb{P}^{1}, Q^{n}\right)$ the orthogonal Grassmannian of lines in $Q^{n}$.

Instead of parametrizing maps $\mathbb{P}^{1} \rightarrow Q^{n}$, it is also of interest to parametrize spaces of rational curves contained in $Q^{n}$. For odd degrees, the latter problem is almost equivalent to Theorem 1] see Corollary 31. However, for even degrees parametrizing spaces of rational curves is much harder and we get full answers in only a few cases; see Theorem 32 and Proposition 34 .

The rest of the paper is devoted to the next case $r=d=2$, which is considerably harder. Thus we aim to understand rational maps

$$
\Phi: \mathbb{P}^{2} \rightarrow Q^{n}:=\left(\begin{array}{c}
\left.q\left(x_{0}, \ldots, x_{n+1}\right)=0\right) \subset \mathbb{P}^{n+1} \\
1
\end{array}\right.
$$


defined by degree 2 homogeneous polynomials $x_{i}=h_{i}(u, v, w) \in k[u, v, w]$. As in Theorem 1, our aim is to describe the moduli spaces $\operatorname{Map}_{2}\left(\mathbb{P}^{2}, Q^{n}\right)$ up to birational equivalence, using $Q^{n}$ and its orthogonal Grassmannians. If $Q^{n}$ is singular then, after a suitable linear coordinate change, we can eliminate some of the variables $x_{i}$ from $q$. Thus from now on $Q^{n}$ denotes a smooth quadric hypersurface of dimension $n$ over a field $k$.

With the exception of Sections 23 we assume form now on that char $k \neq 2$, though this is not always necessary.

In low dimensions we prove in $(37,2),(40,2)$ and $(42,3)$ that

$$
\begin{array}{lll}
\operatorname{Map}_{2}\left(\mathbb{P}^{2}, Q^{1}\right) & \stackrel{\text { bir }}{\sim} & Q^{1} \times \mathbb{P}^{4}, \\
\operatorname{Map}_{2}\left(\mathbb{P}^{2}, Q^{2}\right) & \stackrel{\text { bir }}{\sim} & Q^{2} \times \mathbb{P}^{8}, \\
\operatorname{Map}_{2}\left(\mathbb{P}^{2}, Q^{3}\right) & \stackrel{\text { bir }}{\sim} & \left(Q^{3} \amalg \mathrm{OG}\left(\mathbb{P}^{1}, Q^{3}\right)\right) \times \mathbb{P}^{11} .
\end{array}
$$

Let $\operatorname{Map}_{2}^{\circ}\left(\mathbb{P}^{2}, Q^{n}\right) \subset \operatorname{Map}_{2}\left(\mathbb{P}^{2}, Q^{n}\right)$ denote the open subset consisting of maps that are everywhere defined. If $\Phi \in \operatorname{Map}_{2}^{\circ}\left(\mathbb{P}^{2}, Q^{n}\right)$ and we work over $\mathbb{C}$ then the image of the fundamental class $\Phi_{*}\left[\mathbb{P}^{2}\right]$ is in $H_{4}\left(Q^{n}, \mathbb{Z}\right)$. If $n=2$ (resp. 3 ) then $H_{4}\left(Q^{n}, \mathbb{Z}\right) \cong \mathbb{Z}$ is generated by $Q^{2}$ (resp. $\left.Q^{3} \cap H\right)$ where $H$ denotes the hyperplane class. If $n \geq 5$ then $H_{4}\left(Q^{n}, \mathbb{Z}\right) \cong \mathbb{Z}$ but this time it is generated by the class of any 2plane $L^{2} \subset Q^{n}$. The most interesting case is $n=4$. Then $H_{4}\left(Q^{4}, \mathbb{Z}\right) \cong \mathbb{Z}[A]+\mathbb{Z}[B]$ where $A, B \subset Q^{4}$ are disjoint planes; see [HP47, Book IV, Sec.XIII.4] on linear spaces in quadrics. The same holds over any algebraically closed field if we replace $H_{4}\left(Q^{n}, \mathbb{Z}\right)$ by the group $N_{2}\left(Q^{n}, \mathbb{Z}\right)$ of 2 -cycles modulo numerical equivalence. This suggests that the really interesting case is $n=4$.

The key player in our study is the Veronese surface.

Definition 2. A Veronese surface is the image of the map

$$
\mathbb{P}^{2} \rightarrow \mathbb{P}^{5} \quad \text { given by } \quad(u: v: w) \mapsto\left(u^{2}: v^{2}: w^{2}: u v: v w: u w\right),
$$

composed with any automorphism of $\mathbb{P}^{5}$. They were first studied in Ver1884. Veronese surfaces have numerous exceptional and extremal properties.

- If a surface contains a conic through any 2 of its points then it is a plane, quadric or Veronese; this follows from the Kronecker-Castelnuovo theorem; see Cas1894.

- If the secant lines of a surface cover a subvariety of dimension $\leq 4$, then it is the Veronese (or it is contained in a linear subspace of dimension $\leq 4$ ). This was proved by Severi Sev1901] (see Dal85, for a short proof) and generalized by Kuiper and Pohl Kui62, KP77, to non-algebraic surfaces.

- Veronese surfaces give rise to remarkable birational maps. As pointed out by F. Russo, this seems to have been known to Bertini (see [Ber1907, Secs.XV.9-10] or [Ber24, Secs.XVI.9-10]) and Coble [Cob22, Thm.17], but the earliest explicit mention may be in [SR49, p.188]. A modern treatment is given by Ein and ShepherdBarron ESB89. These connections have been extended by Pirio and Russo to include Jordan algebras PR14. See the book Rus16 for a detailed treatment of many of these results.

We will use a classification of projections and equations of Veronese surfaces; see (41) and (48).

The geometric version of the main result is the following; 
Theorem 3. Let $Q^{4}$ be a smooth quadric 4-fold defined over an algebraically closed field. Then $\operatorname{Map}_{2}\left(\mathbb{P}^{2}, Q^{4}\right)$ has 5 irreducible components, each birational to $\mathbb{P}^{20}$, and $\operatorname{Map}_{2}^{\circ}\left(\mathbb{P}^{2}, Q^{4}\right)$ is dense in $\operatorname{Map}_{2}\left(\mathbb{P}^{2}, Q^{4}\right)$. The 5 components of $\operatorname{Map}_{2}^{\circ}\left(\mathbb{P}^{2}, Q^{4}\right)$ can be described as follows

(1) (Veronese) In these cases $\Phi\left(\mathbb{P}^{2}\right) \subset Q^{4}$ is a Veronese surface. There are 2 such components corresponding to $\Phi_{*}\left[\mathbb{P}^{2}\right]=3[A]+[B]$ and $\Phi_{*}\left[\mathbb{P}^{2}\right]=$ $[A]+3[B]$, where $A, B \subset Q^{4}$ are disjoint planes.

(2) (Projected Veronese) In these cases $\Phi\left(\mathbb{P}^{2}\right) \subset Q^{4}$ is a singular projection of a Veronese surface. There is 1 such component and $\Phi_{*}\left[\mathbb{P}^{2}\right]=2[A]+2[B]$.

(3) (Quadruple plane) In these cases $\Phi\left(\mathbb{P}^{2}\right) \subset Q^{4}$ is a plane. There are 2 such components corresponding to $\Phi_{*}\left[\mathbb{P}^{2}\right]=4[A]$ and $\Phi_{*}\left[\mathbb{P}^{2}\right]=4[B]$.

The corresponding subvarieties of $\operatorname{Map}_{2}^{\circ}\left(\mathbb{P}^{2}, Q^{4}\right)$ will be denoted by $\operatorname{Map}_{V}^{\circ}\left(\mathbb{P}^{2}, Q^{4}\right)$, $\operatorname{Map}_{P V}^{\circ}\left(\mathbb{P}^{2}, Q^{4}\right), \operatorname{Map}_{Q P}^{\circ}\left(\mathbb{P}^{2}, Q^{4}\right)$ and their closures in $\operatorname{Map}_{2}\left(\mathbb{P}^{2}, Q^{4}\right)$ by $\operatorname{Map}_{V}\left(\mathbb{P}^{2}, Q^{4}\right)$, $\operatorname{Map}_{P V}\left(\mathbb{P}^{2}, Q^{4}\right)$ and $\operatorname{Map}_{Q P}\left(\mathbb{P}^{2}, Q^{4}\right)$.

Our main aim is to describe $\operatorname{Map}\left(\mathbb{P}^{2}, Q^{4}\right)$ over arbitrary fields $k$, so Theorem 3 is but a special case of the following 3 results when $k$ is algebraically closed. The most interesting is the description of the Veronese locus; it is proved in Section 5. The easier Propositions 5, 6 are treated in Section 4. See Notation 20 for the terminology involving quadratic forms.

Theorem 4 (Veronese). Let $Q^{4}$ be a smooth quadric 4-fold over a field $k$. Then

$$
\operatorname{Map}_{V}\left(\mathbb{P}^{2}, Q^{4}\right) \stackrel{\text { bir }}{\sim}\{ \pm \sqrt{-\Delta}\} \times Q^{4} \times \mathbb{P}^{16},
$$

where $\Delta$ is the discriminant of $Q^{4}$ and $\{ \pm \sqrt{-\Delta}\}$ denotes the 2-point algebraic set defined by the equation $t^{2}+\Delta=0$ over $k$.

Furthermore, $\operatorname{Map}_{V}^{\circ}\left(\mathbb{P}^{2}, Q^{n}\right)(k)$ is nonempty iff $-\Delta$ is a square in $k$ and $Q^{4}(k) \neq$ $\emptyset$. If these hold then $\operatorname{Map}_{V}\left(\mathbb{P}^{2}, Q^{4}\right)$ is birational to $\mathbb{P}^{20} \amalg \mathbb{P}^{20}$, the disjoint union of 2 copies of $\mathbb{P}^{20}$

The description of the projected Veronese maps is not hard and the quadruple planes are obvious. The following are special cases of [43,2) and (39,1).

Proposition 5 (Projected Veronese). Let $Q^{4}$ be a smooth quadric 4-fold. Then

$$
\operatorname{Map}_{P V}\left(\mathbb{P}^{2}, Q^{4}\right) \stackrel{\text { bir }}{\sim} \mathrm{OG}\left(\mathbb{P}^{1}, Q^{4}\right) \times \mathbb{P}^{15},
$$

where $\mathrm{OG}\left(\mathbb{P}^{1}, Q^{4}\right)$ denotes the orthogonal Grassmannian of lines in $Q^{4}$. Furthermore, $\operatorname{Map}_{P V}^{\circ}\left(\mathbb{P}^{2}, Q^{4}\right)(k)$ is nonempty iff the anisotropic rank of $Q^{4}$ is $\leq 2$ and then $\operatorname{Map}_{P V}\left(\mathbb{P}^{2}, Q^{4}\right)$ is birational to $\mathbb{P}^{20}$.

Proposition 6 (Quadruple plane). Let $Q^{4}$ be a smooth quadric 4-fold. Then

$$
\operatorname{Map}_{Q P}\left(\mathbb{P}^{2}, Q^{4}\right) \stackrel{\text { bir }}{\sim} \mathrm{OG}\left(\mathbb{P}^{2}, Q^{4}\right) \times \mathbb{P}^{17},
$$

where $\mathrm{OG}\left(\mathbb{P}^{2}, Q^{4}\right)$ denotes the orthogonal Grassmannian of 2-planes in $Q^{4}$. Furthermore, $\operatorname{Map}_{Q P}^{\circ}\left(\mathbb{P}^{2}, Q^{n}\right)(k) \neq \emptyset$ iff $Q^{4}$ is split over $k$ and then $\operatorname{Map}_{Q P}\left(\mathbb{P}^{2}, Q^{4}\right)$ is birational to $\mathbb{P}^{20} \amalg \mathbb{P}^{20}$.

Note that if the anisotropic rank of $Q^{4}$ is $\leq 2$ and $-\Delta$ is a square then $Q^{4}$ is split. Thus for non-split forms we have either only 1 irreducible component with smooth $k$-points (if the anisotropic rank is 2) or 2 irreducible components with smooth $k$-points (if $-\Delta$ is a square). 
The above results count maps from a fixed $\mathbb{P}^{2}$ to a quadric $Q^{4}$. Frequently it is more interesting to count maps up to coordinate changes by $\operatorname{Aut}\left(\mathbb{P}^{2}\right)$ or by $\operatorname{Aut}\left(Q^{4}\right)$.

Taking quotient by Aut $\left(\mathbb{P}^{2}\right)$ is essentially the same as working with the components of the Chow variety Chow $\left(Q^{4}\right)$ that parametrize Veronese surfaces (resp. projected Veronese surfaces). Let us denote these by $\operatorname{Chow}_{V}\left(Q^{4}\right)\left(\operatorname{resp} \operatorname{Chow}_{P V}\left(Q^{4}\right)\right)$. Let $H_{5}$ denote the hyperplane class on $\mathbb{P}^{5}$ and let $F_{k} \subset Q_{k}^{4}$ be a surface that is a Veronese surface over $\bar{k}$. Then $\left|2 H_{5}\right|_{F_{k}}+K_{F_{k}} \mid$ is the linear system of lines giving an isomorphism $F_{k} \cong \mathbb{P}_{k}^{2}$. Therefore $\operatorname{Chow}_{V}\left(Q^{4}\right) \times \operatorname{Aut}\left(\mathbb{P}^{2}\right) \stackrel{\text { bir }}{\sim} \operatorname{Map}_{V}\left(\mathbb{P}^{2}, Q^{4}\right)$ and similarly for $\operatorname{Chow}_{P V}\left(Q^{4}\right)$. Hence the previous results determine the stable birational types of $\operatorname{Chow}_{V}\left(Q^{4}\right)$ and $\operatorname{Chow}_{P V}\left(Q^{4}\right)$. The proofs yield the following slightly stronger forms; see [46,6) and [43,1).

Corollary 7 (Chow variety version). Let $Q^{4}$ be a smooth quadric 4-fold over a field $k$. Then

$$
\begin{aligned}
& \operatorname{Chow}_{V}\left(Q^{4}\right) \times \mathbb{P}^{4} \stackrel{\text { bir }}{\sim}\{ \pm \sqrt{-\Delta}\} \times Q^{4} \times \mathbb{P}^{12} \text { and } \\
& \operatorname{Chow}_{P V}\left(Q^{4}\right) \stackrel{\text { bir }}{\sim} \mathrm{OG}\left(\mathbb{P}^{1}, Q^{4}\right) \times \mathbb{P}^{7} \text {. }
\end{aligned}
$$

It is quite likely that one can cancel the factor $\mathbb{P}^{4}$ but my proof does not seem to work without it.

Chow $_{P V}\left(Q^{4}\right)$ is contained in the irreducible component $\operatorname{Chow}_{C I}\left(Q^{4}\right) \subset \operatorname{Chow}\left(Q^{4}\right)$ parametrizing complete intersections of $Q^{4}$ with a hyperplane and a quadric. Another interesting irreducible component of $\operatorname{Chow}\left(Q^{4}\right)$ parametrizing degree 4 surfaces is discussed in (47).

We show in Section 6 that working up to coordinate changes by both $\operatorname{Aut}\left(\mathbb{P}^{2}\right)$ and $\operatorname{Aut}\left(Q^{4}\right)$ yields a very nice answer, especially in the Veronese case.

Theorem 8 (Veronese). Let $k$ be a field. A smooth quadric hypersurface $Q^{4} \subset \mathbb{P}^{5}$ contains a Veronese surface iff its equation can be written as

$$
Q_{a, b}^{4}=\left(x_{0} x_{5}=x_{1}^{2}+a x_{2}^{2}+b x_{3}^{2}+a b x_{4}^{4}\right) .
$$

Up to coordinate changes by $\operatorname{Aut}\left(\mathbb{P}^{2}\right) \times \operatorname{Aut}\left(Q_{a, b}^{4}\right)$, the quadric $Q_{a, b}^{4}$ contains a unique Veronese surface. A representative is given by

$$
(u: v: w) \mapsto\left(u^{2}+a w^{2}: u v: v w: u w: w^{2}: v^{2}+b w^{2}\right) .
$$

Complement 9. Let $k$ be a field. Then $Q_{a, b}^{4} \leftrightarrow\left(a u^{2}+b v^{2}+w^{2}=0\right)$ gives a one-to-one correspondence between

- pairs $\left(V \subset Q^{4}\right)-a$ Veronese surface contained in a smooth quadric hypersurface defined over $k$, up to isomorphism-and

- smooth plane conics $C \subset \mathbb{P}^{2}$ defined over $k$, up to isomorphism.

A proof of this is given in (48,6). Another way of obtaining a conic out of a pair $\left(V \subset Q^{4}\right)$ is as follows. Pick $p \in V$. Intersecting $V$ with the tangent plane of $Q^{4}$ at $p$ gives a hyperplane section of $V$ that is singular at $p$. Since the hyperplane sections of $V$ are conics, we expect that we get a pair of lines and there is a curve $C_{a, b} \subset V$ where this intersection is a double line.

A direct computation using (8,2) (that I did not find illuminating) shows that $C_{a, b}=\left(b u^{2}+a v^{2}+a b w^{2}=0\right)$. We can rewrite this as $b\left(a u_{1}\right)^{2}+a\left(b v_{1}\right)^{2}+a b w^{2}=$ $a b\left(a u_{1}^{2}+b v_{1}^{2}+w^{2}\right)$. 
Remark 10. We can fully diagonalize the quadric $Q_{a, b}^{4}$ by replacing $x_{0}, x_{5}$ by $\frac{1}{2}\left(x_{0}+x_{5}\right)$ and $\frac{1}{2}\left(x_{0}-x_{5}\right)$. Then we end up with the identity

$\frac{1}{4}\left(u^{2}+v^{2}+(a+b) w^{2}\right)^{2}-\frac{1}{4}\left(u^{2}-v^{2}+(a-b) w^{2}\right)^{2}=(u v)^{2}+a(v w)^{2}+b(u w)^{2}+a b\left(w^{2}\right)^{2}$.

Skopenkov pointed out that (if $a, b$ are real and positive) this is equivalent to the quaternionic identity

$$
\frac{1}{4}\left(|A|^{2}+|B|^{2}\right)^{2}-\frac{1}{4}\left(|A|^{2}-|B|^{2}\right)^{2}=|A B|^{2}
$$

for the quaternions $A=u+i w \sqrt{a}$ and $B=v+j w \sqrt{b}$.

The following 2 Propositions are also proved in Section 6

Proposition 11 (Projected Veronese). Let $k$ be a field. A smooth quadric hypersurface $Q^{4} \subset \mathbb{P}^{5}$ contains a projected Veronese surface iff its equation can be written as

$$
Q_{a}^{4}=\left(x_{0} x_{1}+x_{2} x_{3}=x_{4}^{2}+a x_{5}^{2}\right) .
$$

After coordinate changes by $\operatorname{Aut}\left(\mathbb{P}^{2}\right) \times \operatorname{Aut}\left(Q_{a}^{4}\right)$, projected Veronese surfaces can be brought to the form

$$
(u: v: w) \mapsto\left(0: q(u: v: w): u^{2}+a v^{2}: w^{2}: u w: v w\right)
$$

where $\operatorname{deg} q=2$. Restricting $q$ to the line $(w=0)$ gives an isomorphism between the moduli of projected Veronese surfaces in $Q_{a}^{4}$ (up to $\operatorname{Aut}\left(\mathbb{P}^{2}\right) \times \operatorname{Aut}\left(Q_{a}^{4}\right)$ ) and $\operatorname{Sym}^{2}\left(\mathbb{P}^{1}\right) / \mathrm{O}\left(u^{2}+a v^{2}\right)$.

Proposition 12 (Quadruple plane). A smooth quadric hypersurface $Q^{4} \subset \mathbb{P}^{5}$ contains a 2-plane iff its equation can be written as

$$
Q_{\mathrm{split}}^{4}=\left(x_{0} x_{3}+x_{1} x_{4}+x_{2} x_{5}=0\right) .
$$

If char $k \neq 3$ then, after coordinate changes by $\operatorname{Aut}\left(Q_{\mathrm{split}}^{4}\right)$, quadruple planes can be brought to the form

$$
(u: v: w) \mapsto(\partial C / \partial u: \partial C / \partial v: \partial C / \partial w: 0: 0: 0),
$$

where $C=C(u, v, w)$ is a homogeneous cubic. This gives an isomorphism between the moduli of quadruple planes (up to $\operatorname{Aut}\left(\mathbb{P}^{2}\right) \times \operatorname{Aut}\left(Q_{\mathrm{split}}^{4}\right)$ ) and the moduli of plane cubic curves (up to $\operatorname{Aut}\left(\mathbb{P}^{2}\right)$ ).

Next we move to quadrics of dimension $\geq 5$. Note that $\Phi\left(\mathbb{P}^{2}\right)$ is always contained in a 5 -dimensional linear subspace of $\mathbb{P}^{n+1}$, so the cases $n \geq 5$ reduce to the $n \leq$ 4 cases is principle but the parametrization of the solutions for $n \geq 5$ proceeds somewhat differently. The following is shown in Section 7 .

Theorem 13. Assume that $n \geq 5$ and $Q^{n}(k) \neq \emptyset$. Then

$$
\operatorname{Map}_{2}\left(\mathbb{P}^{2}, Q^{n}\right) \stackrel{\text { bir }}{\sim} \operatorname{Sym}^{2}\left(\mathrm{OG}\left(\mathbb{P}^{2}, Q^{n}\right)\right) \times \mathbb{P}^{14},
$$

where $\mathrm{Sym}^{2}$ denotes the symmetric square.

Using 668 2) we can rewrite 131$)$ as

$$
\operatorname{Map}_{2}\left(\mathbb{P}^{2}, Q^{n}\right) \stackrel{\text { bir }}{\sim} \operatorname{Sym}^{2}\left(\mathrm{OG}\left(\mathbb{P}^{1}, Q_{W}^{n-2}\right)\right) \times \mathbb{P}^{2 n+10},
$$

where $Q_{W}^{n-2}$ is the Witt reduction of $Q^{n}$ (see Notation 201). This variant actually shows the structure of $\operatorname{Map}_{2}\left(\mathbb{P}^{2}, Q^{n}\right)$ better than $(13,1)$. 
Remark 14. If $n=4$ then $\{ \pm \sqrt{-\Delta}\} \times Q^{4} \times \mathbb{P}^{2}$ is birational to an irreducible component of $\operatorname{Sym}^{2}\left(\mathrm{OG}\left(\mathbb{P}^{2}, Q^{4}\right)\right.$ ) (or a union of 2 irreducible components if $-\Delta$ is a square in $k$ ) by $(69,4)$. Thus Theorem 13 can be viewed as a generalization of Theorem 4. Note, however that Theorem 13 fails if $Q^{n}(k)=\emptyset$. Indeed, for $n \geq 5$ consider the diagonal quadric $Q^{n}:=\left(x_{0}^{2}+\cdots+x_{n+1}^{2}=0\right)$ over any field $k \subset \mathbb{R}$. Then $\operatorname{Map}_{2}\left(\mathbb{P}^{2}, Q^{n}\right)(k)=\emptyset$ but the conjugate pair of linear spaces

$$
L_{ \pm}^{2}:=\left(x_{0} \pm i x_{1}=x_{2} \pm i x_{3}=x_{4} \pm i x_{5}=x_{6}=\cdots=x_{n+1}=0\right)
$$

shows that $\operatorname{Sym}^{2}\left(\mathrm{OG}\left(\mathbb{P}^{2}, Q^{n}\right) \neq \emptyset\right.$.

The birational equivalence in Theorem[13] is not completely $\operatorname{Aut}\left(Q^{n}\right)$-equivariant. The reason is that we usually imagine the left hand side as $\operatorname{Chow}_{V}\left(Q^{n}\right) \times \operatorname{Aut}\left(\mathbb{P}^{2}\right)$. However, the proof works with pointed Veronese surfaces. Thus it views the left had side as $\operatorname{Univ}_{V}\left(Q^{n}\right) \times \operatorname{Aut}\left(p \in \mathbb{P}^{2}\right)$, where $\operatorname{Univ}_{V}\left(Q^{n}\right) \rightarrow \operatorname{Chow}_{V}\left(Q^{n}\right)$ denotes the universal family of Veronese surfaces in $Q^{n}$ and $\operatorname{Aut}\left(p \in \mathbb{P}^{2}\right)$ is the group of automorphisms of $\mathbb{P}^{2}$ that fix the marked point $p$. We have $\operatorname{Aut}\left(Q^{n}\right)$-equivariance for the latter presentation.

Theorem 13 implies that $\operatorname{Map}_{2}\left(\mathbb{P}^{2}, Q^{n}\right)$ is geometrically irreducible for $n \geq 5$. In particular, projected Veronese surfaces and quadruple planes can be deformed to Veronese surfaces in a 5-dimensional quadric; see Example 55 for explicit descriptions.

I do not know a birational classification of symmetric squares of orthogonal Grassmannians in general. We give an explicit description of $\operatorname{Sym}^{2}\left(\mathrm{OG}\left(\mathbb{P}^{1}, Q^{3}\right)\right)$ in (71) and this implies the following.

Proposition 15. Let $Q^{5}=\left(q\left(x_{0}, \ldots, x_{4}\right)=x_{5} x_{6}\right)$ be a smooth quadric 5-fold over a field $k$. Then

$$
\operatorname{Map}_{2}\left(\mathbb{P}^{2}, Q^{5}\right) \stackrel{\text { bir }}{\sim}\left(\Delta(q) z^{2}=q\left(x_{0}, \ldots, x_{4}\right)\right) \times \mathbb{P}^{22},
$$

where $\Delta(q)$ is the discriminant of $q$.

Note that the quadratic form $\Delta(q) z^{2}-q\left(x_{0}, \ldots, x_{4}\right)$ is never positive definite, thus the Hasse-Minkowski theorem (cf. [Ser73, p.41]) and Theorem 13 imply the following.

Corollary 16. Let $Q^{5}$ be a smooth quadric 5-fold over $k$ such that $Q^{5}(k) \neq \emptyset$. Assume that $k$ is one of the following:

(1) $\mathbb{R}$ or any real closed field,

(2) a number field,

(3) a function field of a curve over a finite field or

(4) a function field of a surface over an algebraically closed field.

Then $\operatorname{Map}_{2}\left(\mathbb{P}^{2}, Q^{5}\right) \stackrel{\text { bir }}{\sim} \mathbb{P}^{26}$.

For other fields the situation is more complicated.

Example 17. Let $K:=k\left(t_{1}, \ldots, t_{4}\right)$ where the $t_{i}$ are algebraically independent. Consider the quadric 5 -fold $Q^{5}:=\left(x_{0}^{2}+t_{1} x_{1}^{2}+\cdots+t_{4} x_{4}^{2}=x_{5} x_{6}\right)$. It is easy to see that

$$
x_{0}^{2}+t_{1} x_{1}^{2}+\cdots+t_{4} x_{4}^{2}=t_{1} t_{2} t_{3} t_{4} \cdot z^{2}
$$

has no solutions in $K$. Therefore, using Theorem 13 and Proposition 15, we obtain that $\operatorname{Sym}^{2}\left(\mathrm{OG}\left(\mathbb{P}^{1}, Q_{W}^{3}\right)\right)(K)=\emptyset$ and hence $\operatorname{Map}_{2}^{\circ}\left(\mathbb{P}^{2}, Q^{5}\right)(K)=\emptyset$. 
Thus the image of any quadratic map $\mathbb{P}^{2} \rightarrow Q^{5}$ is a point or a conic. Examples for the latter are $(u: v: w) \mapsto\left(0: \cdots: 0: u v: u^{2}: t_{4} v^{2}\right)$. These correspond to singular $K$-points of $\operatorname{Map}_{2}\left(\mathbb{P}^{2}, Q^{n}\right)$.

The question of uniqueness of Veronese surfaces $V \subset Q^{n}$ (up to coordinate changes by $\left.\operatorname{Aut}\left(\mathbb{P}^{2}\right) \times \operatorname{Aut}\left(Q^{n}\right)\right)$ is not as simple as in dimension 4 .

Proposition 18. Let $k$ be a field. A smooth quadric hypersurface $Q^{5} \subset \mathbb{P}^{6}$ contains a Veronese surface iff its equation can be written as

$$
Q_{a, b, c}^{5}=\left(x_{0} x_{5}=x_{1}^{2}+a x_{2}^{2}+b x_{3}^{2}+a b x_{4}^{4}+c x_{6}^{2}\right) .
$$

Up to coordinate changes by $\operatorname{Aut}\left(\mathbb{P}^{2}\right) \times \operatorname{Aut}\left(Q_{a, b, c}^{5}\right)$, the quadric $Q_{a, b, c}^{5}$ contains a unique Veronese surface. A representative is given by

$$
(u: v: w) \mapsto\left(u^{2}+a w^{2}: u v: v w: u w: w^{2}: v^{2}+b w^{2}: 0\right) .
$$

The proof is given in (53) and the next examples show that we have nonuniqueness for $n \geq 6$; see also Proposition 24 .

Example 19. If $k$ is algebraically closed then all smooth subquadrics $Q^{4} \subset Q^{n}$ are isomorphic, thus Theorem 8 implies that there is a unique Veronese surface $V \subset Q^{n}$, up to coordinate changes by $\operatorname{Aut}\left(\mathbb{P}^{2}\right) \times \operatorname{Aut}\left(Q^{n}\right)$. The same holds over $\mathbb{R}$ if $Q^{n}$ is the sphere.

However, for most other fields there are quadrics that contain inequivalent Veronese surfaces, up to coordinate changes. Indeed, by Theorem 8, isomorphism classes of quadric 4-folds that contain a Veronese surface are in one-to-one correspondence with isomorphism classes of plane conics. Assume that we have two non-isomorphic ternary forms $x^{2}+a y^{2}+b z^{2}$ and $x^{2}+a y^{2}+c z^{2}$. Consider the quadric 6 -fold

$$
Q_{a, b, c}^{6}=\left(x_{0} x_{7}=x_{1}^{2}+a x_{2}^{2}+b x_{3}^{2}+a b x_{4}^{4}+c x_{5}^{2}+a c x_{6}^{4}\right) .
$$

It contains a Veronese surface $V_{b}$ that satisfies $\left(x_{5}=x_{6}=0\right)$ and also a $V_{c}$ that satisfies $\left(x_{3}=x_{4}=0\right)$. These are inequivalent since the quadrics they span are not isomorphic.

As another example, let $k$ be a number field and consider

$$
Q^{7}:=\left(x_{0}^{2}+x_{1} x_{2}+x_{3} x_{4}+x_{5} x_{6}+x_{7} x_{8}=0\right) .
$$

Note that $a u^{2}-a v^{2} \sim u v$, so every $Q_{a, b}^{4}$ has at least one embedding into $Q^{7}$. Thus there are infinitely many inequivalent embeddings $V \hookrightarrow Q^{7}$ defined over $k$, up to coordinate changes.

Notation 20. From now on, $Q^{n}:=\left(q\left(x_{0}, \ldots, x_{n+1}\right)=0\right) \subset \mathbb{P}^{n+1}$ denotes a smooth quadric hypersurface over a field $k$. Starting with Section 4 we assume that char $k \neq 2$. The explicit formulas are usually worked out only for diagonal forms.

More generally, $Q^{n, i}$ denotes a quadric hypersurface over a field $k$ whose nonsmooth locus has dimension $i$. We use this mainly for $i=0$.

A quadratic form $q$ over $k$-or the corresponding quadric hypersurface $Q$ over $k$-is called isotropic if $Q(k) \neq \emptyset$ and anisotropic if $Q(k)=\emptyset$.

The discriminant of a symmetric bilinear form $q=\sum_{0 \leq i, j \leq n+1} a_{i j} x_{i} x_{j}$ over $k$ is $\Delta(q):=\operatorname{det}\left(a_{i j}\right) \in k /\left(k^{*}\right)^{2}$. This defines the discriminant of any quadratic form whenever char $k \neq 2$. (Note that some authors use different signs and 2-powers.) A quadric $Q^{n}=\left(q\left(x_{0}, \ldots, x_{n+1}\right)=0\right)$ determines the corresponding quadratic form 
$q$ up to a multiplicative scalar $\lambda$. This changes the discriminant by $\lambda^{n+2}$. So the discriminant of $Q^{n}$ is only defined for even dimensional quadrics and then it is

$$
\Delta\left(Q^{n}\right):=\Delta\left(q\left(x_{0}, \ldots, x_{n+1}\right)\right) \in k /\left(k^{*}\right)^{2} .
$$

Note that $Q^{n}$ is smooth iff $\Delta\left(Q^{n}\right) \neq 0$.

For any point $p \in Q^{n}$, let $Q_{p}^{n-1,0} \subset Q^{n}$ denote the intersection of $Q^{n}$ with its tangent plane at $p$. Thus $Q_{p}^{n-1,0}$ is an $(n-1)$-dimensional quadric cone over an $(n-2)$-dimensional smooth quadric - the projectivized tangent cone - denoted by $Q_{p}^{n-2}$. Both $Q_{p}^{n-1,0}$ and $Q_{p}^{n-2}$ are defined over the field $k(p)$. The $Q_{p}^{n-2}$ together form the universal projectivized tangent cone $\operatorname{PTC}\left(Q^{n}\right) \rightarrow Q^{n}$.

Assume next that $Q^{n}(k) \neq \emptyset$. Pick any $p \in Q^{n}(k)$. In suitable coordinates we may assume that $p=(0, \ldots, 0,1)$ and

$$
q\left(x_{0}, \ldots, x_{n+1}\right) \sim q_{p}\left(x_{0}, \ldots, x_{n-1}\right)+x_{n} x_{n+1} .
$$

Then $Q_{p}^{n-2} \cong\left(q_{p}\left(x_{0}, \ldots, x_{n-1}\right)=0\right) \subset \mathbb{P}^{n-1}$. By Theorem 21, $q_{p}$ and $Q_{p}^{n-2}$ are independent of $p$; we denote this quadric by $Q_{W}^{n-2}$ and call it the Witt reduction of $Q^{n}$. We can iterate the Witt reduction until we reach an anisotropic quadric

$$
Q_{\mathrm{an}}=Q_{\mathrm{an}}^{n-2 r}=\left(q_{\mathrm{an}}\left(x_{0}, \ldots, x_{n+1-2 r}\right)=0\right),
$$

called the anisotropic kernel of $Q^{n}$ (or of $q$ ). The number $r$ is called the Witt index. Equivalently, $r-1$ is the maximum dimension of a linear space that is defined over $k$ and contained in $Q^{n}$.

A form is called split if $2 r=n+2$, equivalently, if it can be written as

$$
x_{0} x_{1}+x_{2} x_{3}+\cdots+x_{2 r-2} x_{2 r-1} .
$$

Let $\pi_{p}: Q^{n} \rightarrow \mathbb{P}^{n}$ denote the projection from $p$. We can factor it as the blow-up of $p$ followed by the contraction of the birational transform $E_{p}^{n-1,0}$ of $Q_{p}^{n-1,0}$. Here $E_{p}^{n-1,0}$ is a $\mathbb{P}^{1}$-bundle over $Q_{p}^{n-2} \subset \mathbb{P}^{n}$. This shows that there is an equivalence

$$
\left\{p \in Q^{n}\right\} \leftrightarrow\left\{Q_{W}^{n-2} \subset \mathbb{P}^{n}\right\}
$$

between $n$-dimensional pointed quadrics and $(n-2)$-dimensional quadrics in $\mathbb{P}^{n}$.

A geometric proof of the special case of Witt's theorem that we used above is the following. Fix a point $p_{0} \in Q^{n}(k)$. For any $p \in Q^{n} \backslash Q_{p_{0}}^{n-1,0}$ we get natural isomorphisms (defined over $k(p)$ )

$$
Q_{p}^{n-2} \cong Q_{p}^{n-1,0} \cap Q_{p_{0}}^{n-1,0} \cong Q_{p_{0}}^{n-2} .
$$

This also shows that the universal projectivized tangent cone $\operatorname{PTC}\left(Q^{n}\right) \rightarrow Q^{n}$ is birationally trivial

$$
\operatorname{PTC}\left(Q^{n}\right) \stackrel{\text { bir }}{\sim} Q^{n} \times Q_{W}^{n-2} .
$$

21 (Witt's cancellation theorem). Let $V$ be a vector space and $q$ a non-degenerate quadratic form on $V$. Let $V_{1}, V_{2} \subset V$ be vector subspaces such that $\left(V_{i},\left.q\right|_{V_{i}}\right)$ are non-degenerate and there is an isometry $\phi:\left(V_{1},\left.q\right|_{V_{1}}\right) \rightarrow\left(V_{2},\left.q\right|_{V_{2}}\right)$. One form of Witt's cancellation theorem says that $\phi$ extends to an isometry $\Phi: V \rightarrow V$.

This is one example where the correspondence between quadrics and quadratic forms gets cumbersome. For example, consider $\left(\mathbb{R}^{5}, q:=x_{1}^{2}+x_{2}^{2}+x_{3}^{2}-x_{4}^{2}-x_{5}^{2}\right)$, $V_{1}:=\left(x_{3}=x_{5}=0\right)$ and $V_{2}:=\left(x_{1}=x_{3}=0\right)$. Note that the quadrics $\left(q=x_{3}=\right.$ $\left.x_{5}=0\right)$ and $\left(q=x_{1}=x_{3}=0\right)$ are isomorphic but the quadratic forms $\left(V_{1},\left.q\right|_{V_{1}}\right)$ and $\left(V_{2},\left.q\right|_{V_{2}}\right)$ are not. There is no automorphism of the quadric $(q=0)$ that carries $\left(q=x_{3}=x_{5}=0\right)$ to $\left(q=x_{1}=x_{3}=0\right)$. 
22 (Effective parametrization). The methods of this paper are effective for 4dimensional quadrics, that is, the proofs can be converted into explicit formulas giving all elements of $\operatorname{Map}_{2}\left(\mathbb{P}^{2}, Q^{4}\right)(k)$ for any field $k$, provided we know the answer to some basic questions about $Q^{4}$.

If there is a $k$-map $\phi: \mathbb{P}^{2} \rightarrow Q^{n}$ then $Q^{n}(k) \neq \emptyset$. Conversely, if $p \in Q^{n}(k)$ is any $k$-point then the constant map $\mathbb{P}^{2} \rightarrow\{p\} \hookrightarrow Q^{n}$ is in $\operatorname{Map}_{2}\left(\mathbb{P}^{2}, Q^{n}\right)(k)$. Thus $\operatorname{Map}_{2}\left(\mathbb{P}^{2}, Q^{n}\right)(k) \neq \emptyset$ iff $Q^{n}(k) \neq \emptyset$. To the best of my knowledge, there is no algorithm that decide whether a quadric over an arbitrary field has a $k$-point or not, and we do not claim to say anything about this question.

We show, however, that once we can decide the existence of points, we can also effectively write down all maps in $\operatorname{Map}_{2}\left(\mathbb{P}^{2}, Q^{n}\right)(k)$. To be precise, we need to decide the existence of points also for certain subforms and the condition we need is the following. We start with the cases when $n \leq 5$.

Assumption. For any quadratic form $q\left(x_{0}, \ldots, x_{n+1}\right)$ we are given (or are able to find) a Witt decomposition of $q$. That is, a linear change of coordinates

$$
q\left(x_{0}, \ldots, x_{n+1}\right) \sim q_{\text {an }}\left(y_{0}, \ldots, y_{n-2 r+1}\right)+y_{n-2 r+2} y_{n-2 r+3}+\cdots+y_{n} y_{n+1}
$$

where $q_{\text {an }}$ is anisotropic.

Note that such a linear change of coordinates always exists over any field, but in general we do not know how to find it. Given a field $k$, there is an algorithm to find such a coordinate change for every quadratic form over $k$ iff there is an algorithm that for every quadratic form $q\left(x_{0}, \ldots, x_{r+1}\right)$ over $k$

- either proves that $q$ is anisotropic,

- or finds a non-trivial solution $q\left(a_{0}, \ldots, a_{r+1}\right)=0$.

Thus the assumption is always satisfied of $k$ is algebraically closed or real closed. Over any number field, the Hasse-Minkowski theorem (see, for instance Ser73, p.41]) provides a (very inefficient) algorithm.

For $n \geq 6$ we also need to know a parametrization of degree 2 points of the orthogonal Grassmannian $\mathrm{OG}\left(\mathbb{P}^{2}, Q^{n}\right)$. I do not even know how to do this over nice fields like $\mathbb{Q}$ or $\mathbb{C}(t)$.

\section{Surfaces CONTAining MAny CirCles}

I got interested in these questions after reading the papers SK15, Sko15 which furnish the last step of a project, started by Kummer and Darboux, to describe all surfaces in $\mathbb{R}^{n}$ that contain at least 2 circles through every point; see also Sch01, PSS12.

The inverse of stereographic projection connects this problem with real algebraic surfaces $F$ on the sphere

$$
\mathbb{S}^{n}:=\left(x_{1}^{2}+\cdots+x_{n+1}^{2}=x_{0}^{2}\right) \subset \mathbb{R} \mathbb{P}^{n+1} .
$$

The formulas for stereographic projection are nicest if we project the sphere

$$
\mathbb{S}^{n}:=\left(x_{1}^{2}+\cdots+x_{n}^{2}+z^{2}=1\right)
$$

from the south pole $(0, \ldots, 0,-1)$. Then

$$
\pi\left(x_{1}, \ldots, x_{n}, z\right)=\left(\frac{x_{1}}{1+z}, \ldots, \frac{x_{n}}{1+z}\right)
$$


with inverse

$$
\pi^{-1}\left(x_{1}, \ldots, x_{n}\right)=\left(\frac{2 x_{1}}{1+\Sigma}, \ldots, \frac{2 x_{n}}{1+\Sigma}, \frac{1-\Sigma}{1+\Sigma}\right),
$$

where $\Sigma=\sum_{i} x_{i}^{2}$. A theorem that Ptolemy attributes to Hipparchus $(\sim 190-120$ $\mathrm{BC})$ says that stereographic projection preserves circles; a modern treatment was given by Halley in 1695 .

Any conic on a sphere is a circle, thus describing all surfaces in $\mathbb{R}^{n}$ that contain many circles is equivalent to describing real algebraic surfaces

$$
F \subset \mathbb{S}^{n}:=\left(x_{1}^{2}+\cdots+x_{n+1}^{2}=x_{0}^{2}\right) \subset \mathbb{R P}^{n+1}
$$

that contain many conics.

Building on works of Kac99 and Sch01, the hardest cases are when $F$ is a projection of the Veronese surface or of a degree 8 del Pezzo surface. These are classified in Sko15] for $\mathbb{S}^{4}$. His idea is to rewrite the equation as

$$
x_{1}^{2}+x_{2}^{2}+x_{3}^{2}+x_{4}^{2}=\left(x_{0}-x_{5}\right)\left(x_{0}+x_{5}\right),
$$

think of $x_{1}^{2}+x_{2}^{2}+x_{3}^{2}+x_{4}^{2}$ as the norm of the quaternion $\mathbf{X}:=x_{1}+x_{2} i+x_{3} j+x_{4} k$ and solve the quaternionic equation

$$
\operatorname{norm}(\mathbf{X})=z_{0} z_{5}, \quad \text { where } \quad \mathbf{X} \in \mathbb{H}[u, v], z_{0}, z_{5} \in \mathbb{R}[u, v] .
$$

This is quite nontrivial since $\mathbb{H}[u, v]$ is far from having unique factorization.

In our language, we work with the quadratic form $q:=x_{1}^{2}+\cdots+x_{5}^{2}-x_{0}^{2}$ over $\mathbb{R}$. Its discriminant is $\Delta=-1$ and the anisotropic kernel is $x_{1}^{2}+x_{2}^{2}+x_{3}^{2}+x_{4}^{2}$. Thus we see from Propositions 56 and Theorem 4 that the Veronese components $\operatorname{Map}_{V}\left(\mathbb{P}^{2}, Q^{4}\right)(\mathbb{R}) \stackrel{\text { bir }}{\sim} \mathbb{R P}^{20} \amalg \mathbb{R P}^{20}$ give all real solutions. By Theorem 8 , there is only one solution up to isomorphism. Since a Veronese surface in $\mathbb{P}^{n+1}$ is contained in a linear subspace of dimension $\leq 5$, these imply the following.

Theorem 23. There are precisely 2 surfaces $F \subset \mathbb{S}^{n}$ (up to the group of Möbius transformations Aut $\left.\left(\mathbb{S}^{n}\right)=\mathrm{O}\left(x_{1}^{2}+\cdots+x_{n+1}^{2}-x_{0}^{2}\right)\right)$ that contain a circle through any 2 points $p_{1}, p_{2} \in F$ :

(1) the sphere $\mathbb{S}^{2} \subset \mathbb{S}^{n}$ and

(2) the Veronese surface $\mathbb{R} \mathbb{P}^{2} \hookrightarrow \mathbb{S}^{4} \subset \mathbb{S}^{n}$.

There are several ways to describe the real Veronese surface $\mathbb{R P}^{2} \hookrightarrow \mathbb{S}^{4}$. For example, one can think of the Veronese surface $V$ as the set of rank 1 matrices in the projectivization of the space of $3 \times 3$ symmetric matrices:

$$
(u: v: w) \mapsto\left(\begin{array}{ccc}
u^{2} & u v & u w \\
u v & v^{2} & v w \\
u w & v w & w^{2}
\end{array}\right) .
$$

The identity $(u v)^{2}+(v w)^{2}+(u w)^{2}=u^{2} v^{2}+v^{2} w^{2}+u^{2} w^{2}$ becomes the quadratic equation

$$
x_{01}^{2}+x_{12}^{2}+x_{02}^{2}=x_{00} x_{11}+x_{11} x_{22}+x_{00} x_{22},
$$

which defines an ellipsoid that contains $V$. The right hand side can be diagonalized in a less symmetric form as

$$
\frac{1}{3}\left(x_{00}+x_{11}+x_{22}\right)^{2}-\frac{1}{4}\left(x_{00}-x_{11}\right)^{2}-\frac{1}{12}\left(x_{00}+x_{11}-2 x_{22}\right)^{2} .
$$

This gives an embedding of $\mathbb{R} \mathbb{P}^{2}$ into the standard sphere of radius 1

$$
(u: v: w) \mapsto \frac{\sqrt{3}}{u^{2}+v^{2}+w^{2}}\left(u v: v w: u w: \frac{1}{2}\left(u^{2}-v^{2}\right): \frac{1}{\sqrt{12}}\left(u^{2}+v^{2}-2 w^{2}\right)\right) .
$$


Another form is given in Remark 10 .

$$
(u: v: w) \mapsto \frac{2}{u^{2}+v^{2}+2 w^{2}}\left(u v: v w: u w: w^{2}: \frac{1}{2}\left(u^{2}-v^{2}\right)\right) .
$$

Another form of the Veronese embedding into $\mathbb{R}^{5}$ is obtained by looking at the vector space $V^{(3,3)}$ of $3 \times 3$ symmetric matrices of trace $0 . \mathrm{SO}(3, \mathbb{R})$ acts on $V^{(3,3)}$ by conjugation and the action preserves the eigenvalues. Furthermore, the orbits are in one-to-one correspondence with the ordered set of eigenvalues $\lambda_{1} \leq \lambda_{2} \leq \lambda_{3}$. The generic orbit is large (with stabilizer $(\mathbb{Z} / 2)^{2}$ ) but if 2 eigenvalues coincide, say for $-1,-1,2$, then the orbit is isomorphic to the Veronese embedding of $\mathbb{R} \mathbb{P}^{2}$. The $\mathrm{SO}(3, \mathbb{R})$-invariant sphere containing this Veronese surface is given by the equation $\operatorname{tr}\left(M^{2}\right)=6$.

Note that $M \mapsto M+\mathbf{1}_{3}$ sends a symmetric matrix with eigenvalues $-1,-1,2$ to a symmetric matrix of rank 1 and trace 3 . This shows that the above affine form is equivalent to the projective one.

Other real quadric hypersurfaces can contain more then one families of Veronese surfaces.

Proposition 24. Let $Q^{n} \subset \mathbb{R P}^{n+1}$ be a quadric of dimension $n \geq 4$ and of signature $(r, s)$ where $r \geq s$. Then the number of different Veronese embeddings $\mathbb{R P}^{2} \hookrightarrow Q^{n}$ (up to coordinate changes by $\operatorname{Aut}\left(\mathbb{R P}^{2}\right) \times \operatorname{Aut}\left(Q^{n}\right)$ ) is

(1) zero if $s=0$ or $r \leq 4, s \leq 2$,

(2) one if $r \geq 5$ and $s \in\{1,2\}$ or if $(r, s)=(3,3),(4,3),(4,4)$ and

(3) at most 3 in general.

Proof. If $n=4$ then $Q^{n}$ contains a Veronese surface only when the signature is $(5,1),(1,5)$ or $(3,3)$. Thus we need to enumerate the different solutions to $(r, s)=$ $\left(r^{\prime}, s^{\prime}\right)+\left(r^{\prime \prime}, s^{\prime \prime}\right)$ where $\left(r^{\prime}, s^{\prime}\right)$ is one of $(5,1),(1,5)$ or $(3,3)$. There are clearly at most 3 solutions but fewer is $s$ is small or for occasional symmetries.

It would be quite interesting to understand higher dimensional generalizations of our results. The following form seems to be the most promising.

Problem 25. Classify real algebraic varieties $X \subset \mathbb{S}^{n}$ such that every point pair $x_{1}, x_{2} \in X$ is contained in a circle $C\left(x_{1}, x_{2}\right) \subset X$.

The paper [R10] classifies complex algebraic varieties $X \subset \mathbb{P}^{n+1}$ that are conicconnected, that is, every point pair $x_{1}, x_{2} \in X$ is contained in at least one conic $C\left(x_{1}, x_{2}\right) \subset X$; see also Rus16, Chap.4]. Thus one needs to decide which conicconnected varieties give real examples in $\mathbb{S}^{n}$. There are many conic-connected Fano varieties of Picard number 1 and these are not yet fully understood. However, if $\operatorname{dim} X \leq 6$, then these are all so called Mukai manifolds and a complete classification of Mukai manifolds is given in Muk89, Mel99. Thus the list of [R10 is complete for $\operatorname{dim} X \leq 6$, hence it could be feasible to answer the above problem when $\operatorname{dim} X \leq 6$.

An interesting extremal case is the Veronese embedding of $\mathbb{P}^{n}$ by quadrics. By a theorem of Gallarati (see Rus16, 3.4.4]) if $X^{n} \subset \mathbb{P}^{N}$ is a linearly non-degenerate conic-connected variety and $N \geq \frac{1}{2} n(n+3)$ then $N=\frac{1}{2} n(n+3)$ and $X^{n}$ is the Veronese embedding of $\mathbb{P}^{n}$ by quadrics. See [LP71] for a version for differentiable manifolds. One should study which quadrics contain these higher dimensional Veronese varieties. 


\section{MAPS OF $\mathbb{P}^{1}$ TO QUADRICS}

Here we describe the spaces $\operatorname{Map}_{d}\left(\mathbb{P}^{1}, Q^{n}\right)$ parametrizing degree $d$ maps from $\mathbb{P}^{1}$ to a quadric. Equivalently, we describe solutions of a homogeneous quadratic equation $q\left(x_{0}, \ldots, x_{n+1}\right)=0$, defined over a field $k$, where the $x_{i}=h_{i}(u, v)$ are degree $d$ homogeneous polynomials in 2 variables $u, v$.

The proof of Theorem 1 is a direct consequence of the following 2 propositions.

Proposition 26. Let $Q^{n}$ be a smooth quadric of dimension $n \geq 3$. Then

$$
\operatorname{Map}_{d}\left(\mathbb{P}^{1}, Q^{n}\right) \stackrel{\text { bir }}{\sim} \operatorname{Map}_{d-2}\left(\mathbb{P}^{1}, Q^{n}\right) \times \mathbb{P}^{2 n} \quad \text { for } d \geq 3 .
$$

Proposition 27. Let $Q^{n}$ be a smooth quadric of dimension $n \geq 3$. Then

$$
\operatorname{Map}_{1}\left(\mathbb{P}^{1}, Q^{n}\right) \stackrel{\text { bir }}{\sim} \mathrm{OG}\left(\mathbb{P}^{1}, Q^{n}\right) \times \mathbb{P}^{3} \quad \text { and } \quad \operatorname{Map}_{2}\left(\mathbb{P}^{1}, Q^{n}\right) \stackrel{\text { bir }}{\sim} Q^{n} \times \mathbb{P}^{2 n} .
$$

Proof. The image of a degree 1 map is a line, this gives the formula for $\operatorname{Map}_{1}\left(\mathbb{P}^{1}, Q^{n}\right)$.

Let $\operatorname{Map}_{2}^{\circ}\left(\mathbb{P}^{1}, Q^{n}\right)$ denote those degree 2 maps that map $\mathbb{P}^{1}$ isomorphically onto a conic. By [Kol96, II.3.14], $\operatorname{Map}_{2}^{\circ}\left(\mathbb{P}^{1}, Q^{n}\right)$ is an open and dense subset of $\operatorname{Map}_{2}\left(\mathbb{P}^{1}, Q^{n}\right)$; this can also be seen by a simple dimension count here. A conic is uniquely determined by any 3 of its points, say the images of $0,1, \infty \in \mathbb{P}^{1}$. This gives an open embedding (hence a birational equivalence)

$$
\operatorname{Map}_{2}^{\circ}\left(\mathbb{P}^{1}, Q^{n}\right) \hookrightarrow Q^{n} \times Q^{n} \times Q^{n} .
$$

The birational equivalence $Q^{n} \times Q^{n} \times Q^{n} \stackrel{\text { bir }}{\sim} Q^{n} \times \mathbb{P}^{2 n}$ can be seen as follows. Fix two hyperplanes $H_{0}, H_{1} \subset \mathbb{P}^{n+1}$. Given $p \in Q^{n}$ and $q_{i} \in H_{i}$, there is a unique degree 2 map $\phi: \mathbb{P}^{1} \rightarrow Q^{n}$ such that $\phi(\infty)=p$ and, $\phi(i)$ is the residual intersection point of $\left\langle p, q_{i}\right\rangle \cap Q^{n}$ for $i=0,1$.

Note that $n=2$ is exceptional for Proposition 27 and

$$
\operatorname{Map}_{2}\left(\mathbb{P}^{1}, Q^{2}\right) \stackrel{\text { bir }}{\sim}\left(Q^{2} \times \mathbb{P}^{4}\right) \amalg\left(\mathrm{OG}\left(\mathbb{P}^{1}, Q^{2}\right) \times \mathbb{P}^{5}\right) .
$$

The plan to prove Proposition 26 is the following. Given a degree $d$ map $\phi$ : $\mathbb{P}^{1} \rightarrow Q^{n}$, we aim to write its image as the directrix of a ruled surface $S$. For suitable choice of $S$ the residual intersection of $S$ and $Q^{n}$ is the image of a degree $d-2$ map $\psi: \mathbb{P}^{1} \rightarrow Q^{n}$. We start with a general discussion on ruled surfaces.

28 (Ruled surfaces). Let $C$ be a smooth projective curve and $\phi_{i}: C \rightarrow \mathbb{P}^{n}$ two morphisms. We want to understand the ruled surface swept out by the lines $\left\langle\phi_{1}(p), \phi_{2}(p)\right\rangle$ for $p \in C$.

Consider the abstract ruled surface $S:=\mathbb{P}_{C}\left(\phi_{1}^{*} \mathcal{O}_{\mathbb{P}^{n}}(1)+\phi_{2}^{*} \mathcal{O}_{\mathbb{P}^{n}}(1)\right)$. It has 2 natural disjoint sections $C_{1}, C_{2} \subset S$ and $\left.\mathcal{O}_{S}(1)\right|_{C_{i}} \cong \phi_{i}^{*} \mathcal{O}_{\mathbb{P}^{n}}(1)$. Consider the exact sequence

$$
\left.0 \rightarrow \mathcal{O}_{S}(1)\left(-C_{1}-C_{2}\right) \rightarrow \mathcal{O}_{S}(1) \rightarrow \mathcal{O}_{S}(1)\right|_{C_{1}}+\left.\mathcal{O}_{S}(1)\right|_{C_{2}} \rightarrow 0 .
$$

Since $\mathcal{O}_{S}(1)\left(-C_{1}-C_{2}\right)$ has degree -1 on the rulings, all of its cohomologies are 0 . Thus we get that

$$
H^{0}\left(S, \mathcal{O}_{S}(1)\right) \cong H^{0}\left(C, \phi_{1}^{*} \mathcal{O}_{\mathbb{P}^{n}}(1)\right)+H^{0}\left(C, \phi_{2}^{*} \mathcal{O}_{\mathbb{P}^{n}}(1)\right) .
$$

That is, the pair of morphisms $\left(\phi_{1}, \phi_{2}\right)$ uniquely extends to a morphism $\Phi: S \rightarrow \mathbb{P}^{n}$. The degree of the image, that is, the self-intersection of $\Phi^{*} \mathcal{O}_{\mathbb{P}^{n}}(1)$ is $\operatorname{deg} \phi_{1}+\operatorname{deg} \phi_{2}$. 
We can do better if there are $r$ points $p_{1}, \ldots, p_{r} \in C$ such that $\phi_{1}\left(p_{i}\right)=$ $\phi_{2}\left(p_{i}\right)$ holds. More generally, let $Z \subset C$ be a 0 -dimensional subscheme such that $\left.\phi_{1}\right|_{Z}=\left.\phi_{2}\right|_{Z}$. Let us denote this map by $\phi_{Z}$. We have natural restriction maps $r_{i}: \phi_{i}^{*} \mathcal{O}_{\mathbb{P}^{n}}(1) \rightarrow \phi_{Z}^{*} \mathcal{O}_{\mathbb{P}^{n}}(1)$. We define the sheaf $E$ as the kernel of the map

$$
r_{1}-r_{2}: \phi_{1}^{*} \mathcal{O}_{\mathbb{P}^{n}}(1) \oplus \phi_{2}^{*} \mathcal{O}_{\mathbb{P}^{n}}(1) \rightarrow \phi_{Z}^{*} \mathcal{O}_{\mathbb{P}^{n}}(1) .
$$

Then $E$ is locally free of rank 2 and we have natural surjections $E \rightarrow \phi_{i}^{*} \mathcal{O}_{\mathbb{P}^{n}}(1)$. Now we set $S:=\mathbb{P}_{C}(E)$. As before it has 2 natural sections $C_{1}, C_{2} \subset S$ such that $\left.\mathcal{O}_{S}(1)\right|_{C_{i}} \cong \phi_{i}^{*} \mathcal{O}_{\mathbb{P}^{n}}(1)$, but now $C_{1} \cap C_{2} \cong Z$. The pair of morphisms $\left(\phi_{1}, \phi_{2}\right)$ uniquely extends to a morphism $\phi_{12}: C_{1} \cup C_{2} \rightarrow \mathbb{P}^{n}$. As before, there is a unique extension to $\Phi: S \rightarrow \mathbb{P}^{n}$. The gain is that the self-intersection of $\Phi^{*} \mathcal{O}_{\mathbb{P}^{n}}(1)$ is $\operatorname{deg} \phi_{1}+\operatorname{deg} \phi_{2}-\operatorname{deg} Z$.

Let now $Q^{n} \subset \mathbb{P}^{n+1}$ be a smooth quadric, $C$ a smooth curve and $\phi: C \rightarrow Q^{n}$ a morphism of degree $a$. Choose any other morphism $\psi: C \rightarrow \mathbb{P}^{n+1}$ of degree $b$. We get a ruled surface $\Phi: S(\phi, \psi) \rightarrow \mathbb{P}^{n+1}$. Assume that its image is not contained in $Q^{n}$. Then $\Phi^{-1}\left(Q^{n}\right)$ is in the linear system of sections of $\mathcal{O}_{S}(2)=\Phi^{*} \mathcal{O}_{\mathbb{P}^{n+1}}(2)$. By construction it contains $C_{1}$; let $R:=R(\phi, \psi)$ denote the residual intersection. Thus $R$ is a union of a section $C_{3} \cong C$ of $S$ plus possibly some rulings. These rulings are over those points $p \in C$ for which the line $\langle\phi(p), \psi(p)\rangle$ is contained in $Q^{n}$ and possibly over those points $p \in C$ for which $\phi(p)=\psi(p)$. Thus we get a new morphism

$$
\phi * \psi:\left.\Phi\right|_{C_{3}}: C \rightarrow Q^{n} .
$$

If there is a 0-dimensional subscheme $Z \subset C$ such that $\left.\phi\right|_{Z}=\left.\psi\right|_{Z}$ then the degree of the residual curve $R$ is $2 \operatorname{deg} S-\operatorname{deg} \phi=\operatorname{deg} \phi+2 \operatorname{deg} \psi-2 \operatorname{deg} Z$ hence

$$
\operatorname{deg}(\phi * \psi)=\operatorname{deg} \phi+2 \operatorname{deg} \psi-2 \operatorname{deg} Z-\# \text { (rulings in } R \text { ). 228,2) }
$$

We get an interesting degenerate case if $\psi$ also maps to $Q^{n}$. Then $\Phi^{-1}\left(Q^{n}\right)$ contains both sections $C_{1}, C_{2}$, hence the residual curve $R:=R(\phi, \psi)$ is a union of $\operatorname{deg} \phi+$ $\operatorname{deg} \psi$ rulings.

29 (Proof of (26) ). We aim to prove the birational equivalence

$$
\operatorname{Map}_{d}\left(\mathbb{P}^{1}, Q^{n}\right) \stackrel{\text { bir }}{\sim} \operatorname{Map}_{d-2}\left(\mathbb{P}^{1}, Q^{n}\right) \times \mathbb{P}^{2 n} \text { for } d \geq 3 .
$$

We fix the points $0,1, \infty \in \mathbb{P}^{1}$, these will play a special role in the construction. To emphasize this, we write $\operatorname{Map}_{d}\left(\mathbb{P}^{1}, 0,1, \infty ; Q^{n}\right)$ instead of $\operatorname{Map}_{d}\left(\mathbb{P}^{1}, Q^{n}\right)$, though these spaces are isomorphic.

Fix an auxiliary hyperplane $L^{n} \subset \mathbb{P}^{n+1}$. We construct a morphism

$$
\Pi_{d}: \operatorname{Map}_{d}\left(\mathbb{P}^{1}, 0,1, \infty ; Q^{n}\right) \rightarrow \operatorname{Map}_{d-2}\left(\mathbb{P}^{1}, 0,1, \infty ; Q^{n}\right) .
$$

Let $\phi_{d}:\left(\mathbb{P}^{1}, 0,1, \infty\right) \rightarrow Q^{n}$ be a morphism of degree $d$. Let $\phi_{1}:\left(\mathbb{P}^{1}, 0,1, \infty\right) \rightarrow$ $\mathbb{P}^{n+1}$ be a morphism of degree 1 that sends $0 \mapsto \phi_{d}(0), \infty \mapsto \phi_{d}(\infty)$ and 1 to the intersection of $L^{n}$ with the line $\left\langle\phi_{d}(0), \phi_{d}(\infty)\right\rangle$. As in (28) the pair $\left(\phi_{d}, \phi_{1}\right)$ determines a ruled surface $S_{d-1}$ and by 228,2$) Q^{n} \cap S_{d-1}=\phi\left(\mathbb{P}^{1}\right) \cup R_{d-2}$ where $R_{d-2}$ is a curve of degree $d-2$. We check below that it is an irreducible rational curve for general $\phi_{d}$. Since both $\phi_{d}$ and $\psi_{d-2}$ give sections of $S_{d-1}$, the 3 marked points on the image of $\phi_{d}$ give 3 marked points on the image of $\psi_{d-2}$. This determines $\psi_{d-2}:\left(\mathbb{P}^{1}, 0,1, \infty\right) \rightarrow Q^{n}$.

Next we show that the generic fiber of $\Pi_{d}$ is birational to $\mathbb{P}^{2 n}$. To see this, fix $\psi_{d-2}:\left(\mathbb{P}^{1}, 0,1, \infty\right) \rightarrow Q^{n}$. Pick points $x_{0}, x_{\infty} \in Q^{n}$. Let $\phi_{1}:\left(\mathbb{P}^{1}, 0,1, \infty\right) \rightarrow \mathbb{P}^{n+1}$ be a morphism of degree 1 that sends $0 \mapsto x_{0}, \infty \mapsto x_{\infty}$ and 1 to the intersection 
of $L^{n}$ with the line connecting $x_{0}, x_{\infty}$. As in (28) the pair $\left(\psi_{d-2}, \psi_{1}\right)$ determines a ruled surface $S_{d-1}$. By construction $Q^{n} \cap S_{d-1}=\psi\left(\mathbb{P}^{1}\right) \cup R_{d}$. As before, this determines $\phi_{d}:\left(\mathbb{P}^{1}, 0,1, \infty\right) \rightarrow Q^{n}$. We need to check that the curves $R_{d-2}$ and $R_{d}$ are irreducible for general choices of $\phi$ and $\psi$. For this it is enough to find one particular case where the second construction gives an irreducible curve $R_{d}$; the same set-up then proves the converse too. We start with the degenerate case where $\psi_{d-2}$ maps onto a line $L \subset Q^{n}$.

We can now vary $Q^{n}$ in the linear system $\left|\mathcal{O}_{\mathbb{P}^{n+1}}(2)\right|(-L)$, which is base-point free outside $L$. Bertini theorem gives that for general $Q^{n}$ the residual intersection $R_{d}:=\left(S_{d-1} \cap Q^{n}\right) \backslash L$ is irreducible. Since all smooth quadrics of dimension $n$ are isomorphic over $\bar{k}$, irreducibility can be achieved by fixing $Q^{n}$ and changing $S_{d-1}$.

Note that if $n=2$ and $\phi_{d}$ is a curve of bidegree $(1, d-1)$ on $Q^{2}$ then the resulting $R_{d-1}$ is a union of $d-1$ lines. So one really needs to check that the construction gives an irreducible curve for $n \geq 3$.

\section{RATIONAL CURVES ON QUADRICS}

Instead of looking at degree $d$ maps from $\mathbb{P}^{1}$ to $Q^{n}$, it is also of interest to study geometrically rational curves contained in $Q^{n}$.

Definition 30. Let $X$ be a projective variety and $L$ an ample line bundle on $X$. For $g, d \geq 0$ let $\mathrm{M}_{g}^{\circ}(X, d)$ be the space parametrizing morphisms $\phi: C \rightarrow X$ where

$-C$ is a smooth, irreducible curve of genus $g$,

$-\phi$ is a morphism such that $C \rightarrow \phi(C)$ is birational and

$-\operatorname{deg}_{C} \phi^{*} L=d$.

One can think of $\mathrm{M}_{g}^{\circ}(X, d)$ as a subset of the Chow variety Chow $(X)$ and it is frequently denoted by $\operatorname{RatCurve}_{d}(X)$ Kol96, Sec.II.2].

One can also view $\mathrm{M}_{g}^{\circ}(X, d)$ as an open subset of $\bar{M}_{g}(X, d)$, the moduli space of stable maps of genus $g$ and of degree $d$ [FP97]. For most purposes the latter is the best compactification but for the birational properties of $\mathrm{M}_{g}^{\circ}(X, d)$ the precise compactification does not matter much.

Note that $\operatorname{Map}_{d}\left(\mathbb{P}^{1}, X\right)$ is a $\mathrm{PGL}_{2}$-torsor over $\mathrm{M}_{0}^{\circ}(X, d)$ but this torsor is usually not Zariski-locally trivial. However, if $d=2 e+1$ is odd and $(\phi: C \rightarrow X)$ is in $\mathrm{M}_{0}^{\circ}(X, d)$ then $\psi^{*} L \otimes \omega_{C_{n}}^{e}$ is a degree 1 line bundle on $C$. Thus the universal family over $\mathrm{M}_{0}^{\circ}(X, d)$ is Zariski-locally trivial and we conclude that

$$
\operatorname{Map}_{2 e+1}\left(\mathbb{P}^{1}, X\right) \stackrel{\text { bir }}{\sim} \mathrm{M}_{0}^{\circ}(X, 2 e+1) \times \mathrm{PGL}_{2} \stackrel{\text { bir }}{\sim} \mathrm{M}_{0}^{\circ}(X, 2 e+1) \times \mathbb{P}^{3} .
$$

However, for $d=2 e$ even, there can be (and usually there are) degree $2 e$ rational curves on $X$ without $k$-points. Thus we still get a rational map $\operatorname{Map}_{2 e+1}\left(\mathbb{P}^{1}, X\right) \rightarrow$ $\mathrm{M}_{0}^{\circ}(X, 2 e+1)$ but a typical fiber is a non-trivial principal homogeneous space under $\mathrm{PGL}_{2}$. It seems much harder to connect the birational properties of $\operatorname{Map}_{2 e+1}\left(\mathbb{P}^{1}, X\right)$ and $\mathrm{M}_{0}^{\circ}(X, 2 e+1)$.

We are mostly interested in the spaces $\mathrm{M}_{0}^{\circ}\left(Q^{n}, d\right)$. If $n \geq 3$ then a dense open subset of $\mathrm{M}_{0}^{\circ}\left(Q^{n}, d\right)$ parametrizes smooth rational curves in $Q^{n}$; see [Kol96, II.3.14]. For odd values of $d$, Theorem 1 and $(30,1)$ give the following.

Corollary 31. Let $Q^{n}$ be a smooth quadric of dimension $n \geq 3$. Then

$$
\mathrm{M}_{0}^{\circ}\left(Q^{n}, 2 e+1\right) \times \mathbb{P}^{3} \stackrel{\text { bir }}{\sim} \mathrm{OG}\left(\mathbb{P}^{1}, Q^{n}\right) \times \mathbb{P}^{2 e n+3} .
$$


It is quite likely that the $\mathbb{P}^{3}$ factor can be canceled. This is not hard to see for $d=3$ but the above argument does not establish it.

We are much less successful for even degrees and for other genera; we get a complete description in 4 cases only.

Theorem 32. Let $Q^{n}$ be a smooth quadric of dimension n. Then

(1) $\mathrm{M}_{0}^{\circ}\left(Q^{n}, 2\right) \stackrel{b i r}{\sim} \mathbb{P}^{3 n-3}$ if $n \geq 1$,

(2) $\mathrm{M}_{1}^{\circ}\left(Q^{n}, 4\right) \stackrel{\text { bir }}{\sim} \mathbb{P}^{4 n}$ if $n \geq 2$

(3) $\mathrm{M}_{0}^{\circ}\left(Q^{n}, 4\right) \times \mathbb{P}^{2} \stackrel{\text { bir }}{\sim} \operatorname{Sym}^{2}\left(\mathrm{OG}\left(\mathbb{P}^{1}, Q^{n}\right)\right) \times \mathbb{P}^{n+5}$ if $n \geq 3$.

(4) $\mathrm{M}_{1}^{\circ}\left(Q^{n}, 5\right) \times \mathbb{P}^{6} \stackrel{\text { bir }}{\sim} \mathrm{OG}\left(\mathbb{P}^{1}, Q^{n}\right) \times \mathbb{P}^{5 n+3}$ if $n \geq 3$.

Proof. The first two claims are clear. A general conic in $Q^{n}$ is the intersection of $Q^{n}$ with a 2-plane, so

$$
\mathrm{M}_{0}^{\circ}\left(Q^{n}, 2\right) \stackrel{\text { bir }}{\sim} \operatorname{Grass}\left(\mathbb{P}^{2}, \mathbb{P}^{n+1}\right) \stackrel{\text { bir }}{\sim} \mathbb{P}^{3 n-3} .
$$

Similarly, a general degree 4 elliptic curve in $Q^{n}$ is the intersection of $Q^{n}$ with a 3 -plane and a quadric in it, so

$$
\mathrm{M}_{1}^{\circ}\left(Q^{n}, 4\right) \stackrel{\text { bir }}{\sim} \operatorname{Grass}\left(\mathbb{P}^{3}, \mathbb{P}^{n+1}\right) \times \mathbb{P}^{8} \stackrel{\text { bir }}{\sim} \mathbb{P}^{4 n} .
$$

For (3) and (4) we use degree 4 del Pezzo surfaces and start with the smallest case $n=3$. Pick an auxiliary fixed point $p_{0} \in \mathbb{P}^{4} \backslash Q^{3}$.

Let $C_{4} \subset Q^{3}$ be a degree 4 rational normal curve. Pick a point pair $\left\{p_{1}, p_{2}\right\} \in$ $\operatorname{Sym}^{2}\left(C_{4}\right) \cong \mathbb{P}^{2}$ and set $C_{2}:=Q^{3} \cap\left\langle p_{0}, p_{1}, p_{2}\right\rangle$. Note that $C_{4} \cup C_{2}$ is a degree 6 curve of arithmetic genus 1 . The linear system of quadric sections of $Q^{3}$ that contain $C_{4} \cup C_{2}$ has dimension $13-11=2$. We compute in (33) 1 ) that the base locus of such a pencil is $C_{4}+C_{2}+\ell_{1}+\ell_{2}$. This gives a map

$$
\Pi: \mathrm{M}_{1}^{\circ}\left(Q^{3}, 4\right) \times \mathbb{P}^{2} \rightarrow \operatorname{Sym}^{2}\left(\mathrm{OG}\left(\mathbb{P}^{1}, Q^{3}\right)\right) \text {. }
$$

What are the fibers? Given a pair of lines $\left\{\ell_{1}, \ell_{2}\right\} \in \operatorname{Sym}^{2}\left(\mathrm{OG}\left(\mathbb{P}^{1}, Q^{3}\right)\right)$ pick points $r_{1} \in \ell_{1}, r_{2} \in \ell_{2}$. Set $C_{2}:=Q^{3} \cap\left\langle p_{0}, r_{1}, r_{2}\right\rangle$. Note that $B:=C_{2}+\ell_{1}+\ell_{2}$ is a degree 4 curve of arithmetic genus 0 . Using (33) 2) we see that $h^{0}\left(Q^{3}, \mathcal{O}_{Q^{3}}(2)(-B)\right)=5$ and for any 2 sections of $\mathcal{O}_{Q^{3}}(2)(-B)$, the residual intersection is a $C_{4}$. Thus the fiber of $\Pi$ is given by the choices of $r_{1}, r_{2}$ and the residual $C_{4}$. The latter correspond to an open subset of $\operatorname{Grass}\left(\mathbb{P}^{1}, \mathbb{P}^{4}\right)$ which is birational to $\mathbb{P}^{6}$. Given a pair of lines $\ell_{1}, \ell_{2} \in Q^{3} \subset \mathbb{P}^{4}$, we can project them to a $\mathbb{P}^{2}$ and then the choice of the points $r_{i} \in \ell_{i}$ corresponds to the dual $\mathbb{P}^{2}$. This shows that

$$
\mathrm{M}_{0}^{\circ}\left(Q^{3}, 4\right) \times \mathbb{P}^{2} \stackrel{\text { bir }}{\sim} \operatorname{Sym}^{2}\left(\mathrm{OG}\left(\mathbb{P}^{1}, Q^{3}\right)\right) \times \mathbb{P}^{8} .
$$

Next we consider higher dimensional quadrics. Any $C_{4} \subset Q^{n}$ spans a $\mathbb{P}^{4}$. This defines a map

$$
\mathrm{M}_{0}^{\circ}\left(Q^{n}, 4\right) \rightarrow \operatorname{Grass}\left(\mathbb{P}^{4}, \mathbb{P}^{n+1}\right),
$$

whose fiber over an $L^{4} \in \operatorname{Grass}\left(\mathbb{P}^{4}, \mathbb{P}^{n+1}\right)$ is $\mathrm{M}_{0}^{\circ}\left(L^{4} \cap Q^{n}, 4\right)$. We wrote down above that this is stably birational to the universal family of pairs of lines. This family parametrizes pairs $\left(\left\{\ell_{1}, \ell_{2}\right\}, L^{4}\right)$ where $\ell_{1}, \ell_{2}$ are lines in $L^{4} \cap Q^{n}$. We can also parametrize this by first choosing $\left\{\ell_{1}, \ell_{2}\right\} \in \operatorname{Sym}^{2}\left(\mathrm{OG}\left(\mathbb{P}^{1}, Q^{n}\right)\right)$ and then an $L^{4}$ that contains their span. This corresponds to $\operatorname{Sym}^{2}\left(\mathrm{OG}\left(\mathbb{P}^{1}, Q^{n}\right)\right) \times \mathbb{P}^{n-3}$. Putting these together gives that

$$
\operatorname{Sym}^{2}\left(\mathrm{OG}\left(\mathbb{P}^{1}, Q^{n}\right)\right) \times \mathbb{P}^{n-3} \times \mathbb{P}^{8} \stackrel{\text { bir }}{\sim} \mathrm{M}_{0}^{\circ}\left(Q^{n}, 4\right) \times \mathbb{P}^{2} .
$$


In order to prove (4) we again start with the smallest case $n=3$. The linear system of quadric sections of $Q^{3}$ that contain $E_{5}$ has dimension $13-9=4$. By (33),2), a general pencil of such quadrics gives a residual intersection which is a degree 3 rational curve. This gives a map

$$
\Pi: \mathrm{M}_{1}^{\circ}\left(Q^{3}, 5\right) \times \mathbb{P}^{6} \rightarrow \mathrm{M}_{0}^{\circ}\left(Q^{3}, 3\right) .
$$

The fiber is given by pencils in $\left|\mathcal{O}_{Q^{3}}(2)\right|\left(-C_{3}\right)$ which is birational to $\operatorname{Grass}\left(\mathbb{P}^{1}, \mathbb{P}^{7}\right) \stackrel{\text { bir }}{\sim}$ $\mathbb{P}^{12}$. This proves that

$$
\Pi: \mathrm{M}_{1}^{\circ}\left(Q^{3}, 5\right) \times \mathbb{P}^{6} \stackrel{\text { bir }}{\sim} \mathrm{M}_{0}^{\circ}\left(Q^{3}, 3\right) \times \mathbb{P}^{12} \stackrel{\text { bir }}{\sim} \mathrm{OG}\left(\mathbb{P}^{1}, Q^{3}\right) \times \mathbb{P}^{18},
$$

where the second birational equivalence follows from (31). Extending this to higher dimensions works as before since a general $E_{5} \subset Q^{n}$ spans a $\mathbb{P}^{4}$. This defines a map

$$
\mathrm{M}_{1}^{\circ}\left(Q^{n}, 5\right) \rightarrow \operatorname{Grass}\left(\mathbb{P}^{4}, \mathbb{P}^{n+1}\right)
$$

whose fiber over an $L^{4} \in \operatorname{Grass}\left(\mathbb{P}^{4}, \mathbb{P}^{n+1}\right)$ is $\mathrm{M}_{1}^{\circ}\left(L^{4} \cap Q^{n}, 5\right)$.

33 (Curves on degree 4 del Pezzo surfaces). Let $C \subset S_{4}$ be a reduced curve of degree $d$ and arithmetic genus $p_{a}$ on a degree 4 del Pezzo surface $S=S_{4}$. Since

$$
H^{0}\left(S, \mathcal{O}_{S}(2)\right)=13 \text { and } H^{0}\left(C,\left.\mathcal{O}_{S}(2)\right|_{C}\right) \leq 2 d+1,
$$

we see that if $2 d+1 \leq 12$ then we get another effective curve $C^{\prime} \subset S$ such that $C+C^{\prime} \sim \mathcal{O}_{S}(2)$. The adjunction formula $2 p_{a}(C)-2=C(C-H)$ gives that $\left(C^{2}\right)=d+2 p_{a}-2$. From this we get that the degree of $C^{\prime}$ is $d^{\prime}=8-d$ and its arithmetic genus is $p_{a}^{\prime}=4-d+p_{a}$. We used some special cases of this.

(33) 1) If $d=6$ and $p_{a}=1$ then $d^{\prime}=2$ and $p_{a}^{\prime}=-1$. Thus $C^{\prime}$ is the disjoint union of 2 lines. Conversely, if $d=2$ and $p_{a}=-1$ then $\left|C^{\prime}\right|$ is a 6-dimensional linear system whose general member is a smooth elliptic curve.

(33) 2) If $d=5$ and $p_{a}=1$ then $d^{\prime}=3$ and $p_{a}^{\prime}=0$. Thus $\left|C^{\prime}\right|$ is a 4-dimensional linear system whose general member is a smooth rational curve of degree 3 . Conversely, if $d=3$ and $p_{a}=0$ then $\left|C^{\prime}\right|$ is a 5-dimensional linear system whose general member is a smooth elliptic curve.

(33) 3) If $d=4$ and $g=0$ then $d^{\prime}=4$ and $g^{\prime}=0$. Thus $\left|C^{\prime}\right|$ is a 3-dimensional linear system whose general member if a smooth rational curve.

(33.4) If $d=6$ and $g=0$ then we formally get that $d^{\prime}=2$ and $g^{\prime}=-2$ but usually there is no such curve $C^{\prime}$. We need to understand this case somewhat differently.

Note that $\left(C^{2}\right)=4$, thus $|C|$ maps $S_{4}$ onto a degree 4 surface in $\mathbb{P}^{5}$. There are 2 such surfaces over an algebraically closed field.

One is the Veronese, that is, $\mathbb{P}^{2}$ embedded by $\left|\mathcal{O}_{\mathbb{P}^{2}}(2)\right|$. This represents $S_{4}$ as $\mathbb{P}^{2}$ blown up at 5 points. Note that in this case twice the conic through the 5 points gives the residual curve $C^{\prime}$. This is a non-generic situation.

The other surface is $\mathbb{P}^{1} \times \mathbb{P}^{1}$ embedded by $\left|\mathcal{O}_{\mathbb{P}^{1} \times \mathbb{P}^{1}}(1,2)\right|$. This represents $S_{4}$ as $\mathbb{P}^{1} \times \mathbb{P}^{1}$ blown up at 4 points and $|C|$ is the birational transform of $\left|\mathcal{O}_{\mathbb{P}^{1} \times \mathbb{P}^{1}}(1,2)\right|$.

Working with degree 4 del Pezzo surfaces as in (32) allows us to describe a few more spaces of maps to $Q^{3}$. In all these cases the curves are not linearly normal in $\mathbb{P}^{4}$, so these results do not yield descriptions for higher dimensional quadrics.

Proposition 34. Let $Q^{3}$ be a smooth quadric. Then

(1) $\mathrm{M}_{0}^{\circ}\left(6, Q^{3}\right) \times \mathbb{P}^{3} \stackrel{\text { bir }}{\sim} \mathbb{P}^{21}$ and 
(2) $\mathrm{M}_{1}^{\circ}\left(6, Q^{3}\right) \stackrel{\text { bir }}{\sim} \operatorname{Sym}^{2}\left(\mathrm{OG}\left(\mathbb{P}^{1}, Q^{3}\right)\right) \times \mathbb{P}^{12}$.

Proof. The elliptic case is easier here. Let $E_{6} \subset Q^{3}$ be a general, smooth curve of genus 1. Computing as in the proof of (32) we see that $E_{6}$ is contained in a pencil of quadric sections of $Q^{3}$ by (33) 1). Intersecting any 2 of them gives a pair of lines as the residual intersection curve. This gives a map $\mathrm{M}_{1}^{\circ}\left(6, Q^{4}\right) \rightarrow \operatorname{Sym}^{2}\left(\mathrm{OG}\left(\mathbb{P}^{1}, Q^{3}\right)\right)$.

In order to understand the fiber, note that a given pair of disjoint lines $\ell_{1}+\ell_{2}$ is contained in a $\mathbb{P}^{7}$-of quadrics hence in a $\mathbb{P}^{12}$ of pencils of quadrics. The residual intersections give degree 6 curves of genus 1 . This shows (2).

Next let $C_{6} \subset Q^{3}$ be a general, smooth, rational curve. As we checked in (33. 4), $C_{6}$ lies on a unique degree 4 del Pezzo surface $S$ and on it $\left|C_{6}\right|$ is a linear system mapping onto a surface $P$ that is geometrically isomorphic to $\mathbb{P}^{1} \times \mathbb{P}^{1}$. Note, however, that $\left|C_{6}\right|$ corresponds to $\left|\mathcal{O}_{\mathbb{P}^{1} \times \mathbb{P}^{1}}(1,2)\right|$. Thus, over $k, P \cong \mathbb{P}^{1} \times Q^{2}$ where $Q^{2}$ is a conic in some $\mathbb{P}^{2}$ and $\left|C_{6}\right|$ corresponds to $\pi_{1}^{*} \mathcal{O}_{\mathbb{P}^{1}}(1) \otimes \pi_{2}^{*} \mathcal{O}_{\mathbb{P}^{2}}(1)$ where $\pi_{i}: P \rightarrow \mathbb{P}^{i}$ are the coordinate projections. Let us now consider

$$
\left|\mathcal{O}_{\mathbb{P}^{1}}(1)\right| \times\left|\mathcal{O}_{\mathbb{P}^{1}}(2)\right| \subset\left|\pi_{1}^{*} \mathcal{O}_{\mathbb{P}^{1}}(1) \otimes \pi_{2}^{*} \mathcal{O}_{\mathbb{P}^{2}}(1)\right| .
$$

Geometrically, a general member of $\left|\mathcal{O}_{\mathbb{P}^{1}}(1)\right| \times\left|\mathcal{O}_{\mathbb{P}^{1}}(2)\right|$ consists of 3 lines. On $S_{4}$ these become 3 conics $B_{1}+B_{2}+B_{3}$ where $B_{2}$ is defined over $k$ and $B_{1}, B_{3}$ are disjoint conjugates. So a general point in $\mathrm{M}_{0}^{\circ}\left(6, Q^{3}\right) \times \mathbb{P}^{3}$ is represented by $\left(C_{6}, B_{1}+B_{2}+B_{3}\right)$.

In order to build this space from the other direction, we first pick $B_{2}$, then a pair of points $\left\{p_{1}, p_{3}\right\}$ on $B_{2}$ and after that a pair of conics through $p_{1}, p_{3}$. These choices involve Grass $\left(\mathbb{P}^{2}, \mathbb{P}^{4}\right), \operatorname{Sym}^{2}\left(Q^{2}\right) \cong \mathbb{P}^{2}$ and then (the Weil restriction of) $\operatorname{Grass}\left(\mathbb{P}^{1}, \mathbb{P}^{3}\right)$ which parametrizes pairs of 2 -planes containing $\left\{p_{1}, p_{2}\right\}$. Together these give $\mathbb{P}^{16}$. Finally note that $B_{1}+B_{2}+B_{3}$ is a curve of degree 6 and genus 0 . By (33. 3), a generic $B_{1}+B_{2}+B_{3}$ lies on a unique degree 4 del Pezzo surface $S$ and on it $\left|B_{1}+B_{2}+B_{3}\right|$ is a 5 -dimensional linear system whose general member is a degree 6 smooth rational curve.

One can try to use K3 surfaces in a similar way. This leads to some interesting examples but nothing very useful for our present purposes.

35 (Residual intersections on K3 surfaces). It was observed by Kov94 that if a K3 surface contains a smooth rational curve then usually it also contains another one. Computing the class of the other curve gives some interesting examples.

$\left(C_{9} \subset Q^{3}\right) . \quad H^{0}\left(Q^{3}, \mathcal{O}_{Q^{3}}(3)\right)$ has dimension $\left(\begin{array}{l}7 \\ 4\end{array}\right)-5=30$. Thus a general $C_{9} \subset Q^{3}$ is contained in a unique pencil of $\left|\mathcal{O}_{Q^{3}}(3)\right|$. The intersection of any two members gives a curve $C_{9}+C_{9}^{\prime}$ where $C_{9}^{\prime}$ is another degree 9 rational curve meeting $C$ in 29 points. In fact, $C_{9}^{\prime}$ is the unique degree 9 rational curve in $\mathbb{P}^{4}$ meeting $C$ in 29 points. Indeed, any such rational curve $C_{9}^{*}$ would meet $Q^{3}$ in $29>2 \cdot 9$ points, thus $C_{9}^{*}$ is contained in $Q^{3}$. Next, $C_{9}^{*}$ would meet any member of the pencil $\left|\mathcal{O}_{Q^{3}}(3)\right|\left(-C_{9}\right)$ in at least $29>3 \cdot 9$ points, thus $C_{9}^{*}$ is contained in the base locus of $\left|\mathcal{O}_{Q^{3}}(3)\right|\left(-C_{9}\right)$, which is exactly $C_{9} \cup C_{9}^{\prime}$.

This gives a natural involution on $\mathrm{M}_{0}^{\circ}\left(Q^{3}, 9\right)$.

$\left(C_{8} \subset Q^{3}\right)$. Computing as above we see that a general $C_{8}$ is contained in a $\mathbb{P}^{5}$ of K3 surfaces of degree 6 . Following the method of Kov94 gives that a general $\mathrm{K} 3$ surface contains another smooth rational curve that is linearly equivalent to $39 H-14 C_{8}$. Thus it has degree 200 and meets $C_{8}$ in 262 points. 


\section{Degenerate maps of $\mathbb{P}^{2}$}

Let $\Phi \in \operatorname{Map}_{2}\left(\mathbb{P}^{2}, Q^{n}\right)$ be given by its coordinate functions $h_{0}, \ldots, h_{n+1}$. The $h_{i}$ are in the 6-dimensional vector space $V^{6}$ of degree 2 homogeneous polynomials in 3 variables. We say that $\Phi$ is non-degenerate if the $h_{i}$ span $V^{6}$ and degenerate otherwise. Thus the image of a degenerate map lies in a (possibly singular) subquadric $Q^{r} \subset Q^{n}$ of dimension $\leq 3$. In this section we describe degenerate maps $\mathbb{P}^{2} \rightarrow Q^{n}$, based on their image.

36 (Expected dimension of $\operatorname{Map}_{2}\left(\mathbb{P}^{2}, Q^{n}\right)$ ). A map $\Phi: \mathbb{P}^{2} \rightarrow Q^{n}$ is a map $\Phi$ : $\mathbb{P}^{2} \longrightarrow \mathbb{P}^{n+1}$ whose image lies in $Q^{n}$. Quadratic maps from $\mathbb{P}^{2}$ to $\mathbb{P}^{n+1}$ are given $n+2$ quadratic forms

$$
h_{0}(u, v, w), \ldots, h_{n+1}(u, v, w) \in H^{0}\left(\mathbb{P}^{2}, \mathcal{O}_{\mathbb{P}^{2}}(2)\right) .
$$

We exclude the case when all of the $h_{i}$ are identically 0 and $\left(h_{0}, \ldots, h_{n+1}\right)$ is identified with $\left(\lambda h_{0}, \ldots, \lambda h_{n+1}\right)$ for any nonzero constant $\lambda$. Thus these maps naturally form a projective space of dimension $6(n+2)-1=6 n+11$. The image lies on $Q^{n}$ iff

$$
q\left(h_{0}(u, v, w), \ldots, h_{n+1}(u, v, w)\right) \equiv 0 .
$$

The left hand side is a degree 4 homogeneous polynomial in $u, v, w$ whose coefficients are degree 2 polynomials in the coefficients of the $h_{i}$. Thus (36,2) is equivalent to $\left(\begin{array}{l}6 \\ 2\end{array}\right)=15$ quadratic equations in the coefficients of the $h_{i}$. Thus we have a natural realization

$$
\operatorname{Map}_{2}\left(\mathbb{P}^{2}, Q^{n}\right) \subset \mathbb{P}^{6 n+11}
$$

as the common zero set of 15 quadratic equations. In particular,

$$
\operatorname{dim} \operatorname{Map}_{2}\left(\mathbb{P}^{2}, Q^{n}\right) \geq 6 n+11-15=6 n-4
$$

More precisely, every irreducible component of $\operatorname{Map}_{2}\left(\mathbb{P}^{2}, Q^{n}\right)$ has dimension $\geq 6 n-$ 4. We will see that equality holds if $n \geq 3$.

37 (Maps with small dimensional image). Maps with 0-dimensional image are parametrized by $Q^{n}$.

Maps with 1-dimensional image are obtained as composites $\mathbb{P}^{2} \rightarrow \mathbb{P}^{1} \rightarrow Q^{n}$. The composite can be a quadratic map in two ways. If $\mathbb{P}^{1} \rightarrow Q^{n}$ is linear then $\mathbb{P}^{2} \rightarrow \mathbb{P}^{1}$ should be a pencil of conics, giving a moduli space that is birational to $\operatorname{Map}_{1}\left(\mathbb{P}^{1}, Q^{n}\right) \times \mathbb{P}^{8}$. If $\mathbb{P}^{1} \rightarrow Q^{n}$ is quadratic then $\mathbb{P}^{2} \rightarrow \mathbb{P}^{1}$ should be a pencil of lines, giving a moduli space that is birational to $\operatorname{Map}_{2}\left(\mathbb{P}^{1}, Q^{n}\right) \times \mathbb{P}^{2}$. Using Theorem 1. the spaces of maps whose image is a line (resp. conic) are further birational to

$$
\begin{aligned}
& \operatorname{Map}_{L}\left(\mathbb{P}^{2}, Q^{n}\right) \stackrel{\text { bir }}{\sim} \operatorname{OG}\left(\mathbb{P}^{1}, Q^{n}\right) \times \mathbb{P}^{11} \text { and } \\
& \operatorname{Map}_{C}\left(\mathbb{P}^{2}, Q^{n}\right) \stackrel{\text { bir }}{\sim} Q^{n} \times \mathbb{P}^{2 n+2} .
\end{aligned}
$$

In particular, if $n=1$ and there is a map $\mathbb{P}^{2} \rightarrow Q^{1}$ then $Q^{1}(k) \neq \emptyset$, thus we can write $q \sim x^{2}-y z$. The simplest solution is the inverse of the projection from $(0,1,0)$. More generally, we get solutions of the form

$$
\begin{aligned}
h_{x}(u, v, w) & =\ell_{1}(u, v, w) \ell_{2}(u, v, w), \\
h_{y}(u, v, w) & =\alpha \ell_{1}^{2}(u, v, w), \\
h_{z}(u, v, w) & =\alpha^{-1} \ell_{2}^{2}(u, v, w) .
\end{aligned}
$$


38 (Quadric cone image). Assume that the image of $\Phi: \mathbb{P}^{2} \rightarrow Q^{n}$ is a singular quadric surface. Let $p \in Q^{n}$ be the singular point of $\Phi\left(\mathbb{P}^{2}\right)$. Composing with projection from $p$ we get a map $\pi_{p} \circ \Phi: \mathbb{P}^{2} \rightarrow Q_{p}^{n-2}$ whose image is a conic. By [37.1) the later form a space birational to $Q_{p}^{n-2} \times \mathbb{P}^{2 n-2}$. Choosing $p \in Q^{n}$ and $p^{\prime} \in Q_{p}^{n-2}$ is the same as choosing the line $\ell=\left\langle p, p^{\prime}\right\rangle \in \mathrm{OG}\left(\mathbb{P}^{1}, Q^{n}\right)$ and the point $p \in \ell$. Thus the maps $\pi_{p} \circ \Phi$ are parametrized by $\mathrm{OG}\left(\mathbb{P}^{1}, Q^{n}\right) \times \mathbb{P}^{2 n-1}$. Lifting a map $\phi^{1}: \mathbb{P}^{2} \rightarrow Q^{1}=\left(q\left(x_{0}, x_{1}, x_{2}\right)=0\right) \subset \mathbb{P}^{2}$ to a map to the corresponding quadric cone $\phi^{2,0}: \mathbb{P}^{2} \rightarrow Q^{2,0}=\left(q\left(x_{0}, x_{1}, x_{2}\right)=0\right) \subset \mathbb{P}^{3}$ is equivalent to choosing an arbitrary 3rd component $\phi_{3}^{2,0}(u, v, w)$. Thus the space of maps with quadric cone image is birational to

$$
\operatorname{Map}_{Q C}\left(\mathbb{P}^{2}, Q^{n}\right) \stackrel{\text { bir }}{\sim} \mathrm{OG}\left(\mathbb{P}^{1}, Q^{n}\right) \times \mathbb{P}^{2 n+5}
$$

39 (Quadruple planes). Here we consider the cases when the image of $\Phi\left(\mathbb{P}^{2}\right)$ is a linear subspace $L^{2} \subset Q^{n}$. Thus, in suitable coordinates $\Phi$ is given as $\left(h_{0}, h_{1}, h_{2}, 0, \ldots, 0\right)$. These maps are parametrized by $h_{0}, h_{1}, h_{2} \in H^{0}\left(\mathbb{P}^{2}, \mathcal{O}_{\mathbb{P}^{2}}(2)\right)$, up to a multiplicative constant. For general choices $h_{0}, h_{1}, h_{2}$ have no common zero and then $\Phi$ is an everywhere defined degree 4 morphism $\mathbb{P}^{2} \rightarrow L^{2}$. If $n=4$ then $\Phi_{*}\left[\mathbb{P}^{2}\right]$ equals $4[A]$ or $4[B]$ and every deformation again has the same class. If the $h_{i}$ have common zeros, we get maps of lower degree and even maps whose image is a conic, a line or a point.

All 2-planes in $Q^{n}$ are parametrized by the orthogonal Grassmannian $\mathrm{OG}\left(\mathbb{P}^{2}, Q^{n}\right)$. As we noted before Corollary 7 , the universal family over $\mathrm{OG}\left(\mathbb{P}^{2}, Q^{n}\right)$ is birationally trivial. Thus

$$
\operatorname{Map}_{Q P}\left(\mathbb{P}^{2}, Q^{n}\right) \stackrel{\text { bir }}{\sim} \mathrm{OG}\left(\mathbb{P}^{2}, Q^{n}\right) \times \mathbb{P}^{17}
$$

If $n=4$ then there is a 2-plane defined over $k$ iff $q$ is split and in this case $\mathrm{OG}\left(\mathbb{P}^{2}, Q^{4}\right) \cong \mathbb{P}^{3} \amalg \mathbb{P}^{3}$, hence $\operatorname{Map}_{2}\left(\mathbb{P}^{2}, Q^{4}\right)$ is birational to the disjoint union of 2 copies of $\mathbb{P}^{20}$.

40 (Smooth quadric surface image). If $Q^{2}$ is split then it is isomorphic to $\mathbb{P}^{1} \times \mathbb{P}^{1}$ and quadratic maps $\mathbb{P}^{2} \rightarrow \mathbb{P}^{1} \times \mathbb{P}^{1}$ correspond to a pair of linear projections $\mathbb{P}^{2}-\rightarrow \mathbb{P}^{1}$. If $Q^{2}=x y-z t$ then these are given by

$$
\begin{aligned}
& h_{x}(u, v, w)=\ell_{1}(u, v, w) \ell_{3}(u, v, w), \\
& h_{y}(u, v, w)=\ell_{2}(u, v, w) \ell_{4}(u, v, w), \\
& h_{z}(u, v, w)=\ell_{1}(u, v, w) \ell_{4}(u, v, w), \\
& h_{t}(u, v, w)=\ell_{2}(u, v, w) \ell_{3}(u, v, w) .
\end{aligned}
$$

Each of the pairs $\left(\ell_{1}, \ell_{2}\right)$ and $\left(\ell_{3}, \ell_{4}\right)$ is determined up to a multiplicative scalar, so $\operatorname{Map}_{2}\left(\mathbb{P}^{2}, Q^{2}\right) \stackrel{\text { bir }}{\sim} \mathbb{P}^{10}$

Geometrically, blow up the 2 points $\left(\ell_{1}=\ell_{2}\right)$ and $\left(\ell_{3}=\ell_{4}\right)$ and contract the line $L$ connecting them to a point $p \in Q^{2}$. Thus $\Phi^{-1}$ is given by projecting $Q^{2}$ from $p$ to $\mathbb{P}^{2}$ and composing it with an automorphism of $\mathbb{P}^{2}$.

Thus if the image of $\phi: \mathbb{P}^{2} \rightarrow Q^{n}$ is a smooth quadric surface then $\phi$ can be uniquely obtained by composing the inverse of a projection $\pi_{p}: Q^{n} \rightarrow \mathbb{P}^{n}$ with a linear embedding $\mathbb{P}^{2} \rightarrow \mathbb{P}^{n}$. These unprojection maps form a variety

$$
\operatorname{Map}_{U P}\left(\mathbb{P}^{2}, Q^{n}\right) \stackrel{\text { bir }}{\sim} Q^{n} \times \mathbb{P}^{3 n+2}
$$

In particular, for a quadric surface $Q^{2}$, if there is a map $\mathbb{P}^{2} \rightarrow Q^{2}$ then $Q^{2}(k) \neq \emptyset$. This holds iff $q \sim x^{2}-a y^{2}-z t$. The inverse of the projection from $(0,0,1,0)$ gives 
the obvious solution (uw, $\left.v w, u^{2}-a v^{2}, w^{2}\right)$. More generally, if we write $h_{x}(u, v, w) \pm$ $\sqrt{a} h_{y}(u, v, w)$ as products of linear factors

$$
h_{x} \pm \sqrt{a} h_{y}=\left(\ell_{1} \pm \sqrt{a} \ell_{2}\right)\left(\ell_{3} \pm \sqrt{a} \ell_{4}\right)
$$

we get the general solutions

$$
h_{x}=\ell_{1} \ell_{3}+a \ell_{2} \ell_{4}, h_{y}=\ell_{1} \ell_{4}+\ell_{2} \ell_{3}, h_{z}=\ell_{1}^{2}-a \ell_{2}^{2}, h_{t}=\ell_{3}^{2}-a \ell_{4}^{2},
$$

but some non-obvious changes of $\ell_{1}, \ldots, \ell_{4}$ result in the same map.

Before we go further, we need to recall some classical facts about the Veronese surface; see [Ber1907, Cap.XV], [Ber24, Kap.XVI] or [SR49, Sec.VII.3] for details.

41 (Veronese surface and its projections). Let $\Phi: \mathbb{P}^{2} \rightarrow \mathbb{P}^{n+1}$ be a map given by degree 2 homogeneous polynomials. Since the space of degree 2 homogeneous polynomials in 3 variables has dimension $6, \Phi\left(\mathbb{P}^{2}\right)$ is always contained in a 5 dimensional linear subspace of $\mathbb{P}^{n+1}$. Moreover, up to $\operatorname{Aut}\left(\mathbb{P}^{5}\right)$, there is a unique embedding $\Phi: \mathbb{P}^{2} \rightarrow \mathbb{P}^{5}$ given by degree 2 homogeneous polynomials whose image is not contained in any hyperplane. Its image is called a Veronese surface; we denote it by $V \subset \mathbb{P}^{5}$. A typical example is

$$
\Phi:(u: v: w) \mapsto\left(u^{2}: v^{2}: w^{2}: u v: v w: u w\right) .
$$

For every other quadratic map $\Phi^{\prime}: \mathbb{P}^{2} \rightarrow \mathbb{P}^{n+1}$, we can think of $\Phi^{\prime}\left(\mathbb{P}^{2}\right)$ as a projection of the Veronese surface.

If we think of the dual $\left(\mathbb{P}^{5}\right)^{\vee}$ as the space of conics in $p^{2}$, then we see that $\operatorname{Aut}\left(\mathbb{P}^{2}\right)$ acts on $\mathbb{P}^{5}$ with 3 orbits, corresponding to the rank if the conic. The rank 1 orbit is the Veronese surface, the rank 2 orbit is its secant variety and the rank 3 orbit is open.

This makes it easy to determine the projections of $V$ to $\mathbb{P}^{4}$ : we just need to compute one example for each of the 3 orbits.

- If we project from a point $p$ not on the secant variety, the projection is an isomorphism and we get a smooth degree 4 surface $V_{1}$. For example, projecting from $(1: 1: 1: 0: 0: 0: 0)$ gives

$$
(u: v: w) \mapsto\left(u^{2}-w^{2}: v^{2}-w^{2}: u v: v w: u w\right) .
$$

We see that $V_{1}$ does not lie on any quadric 3 -fold $Q^{3}$.

- If we project from a point on the secant variety but not on $V$, the projection is a degree 4 surface with a double line $V_{2}$. In suitable coordinates it is the image of the map

$$
(u: v: w) \mapsto\left(u^{2}: v^{2}: w^{2}: u v: v w\right)
$$

It is contained in the family of quadrics $\lambda\left(x_{0} x_{1}-x_{4}^{2}\right)+\mu\left(x_{1} x_{2}-x_{5}^{2}\right)$. All of these are singular, namely at the point $(\mu: 0:-\lambda: 0: 0)$.

Thus $V_{2}$ is a complete intersection of any 2 quadrics that contain it.

- If we project from a point on $V$, the projection is a degree 3 surface $V_{3}$. In suitable coordinates it is the image of the map $\mathbb{P}^{2} \rightarrow \mathbb{P}^{4}$ given by

$$
(u: v: w) \mapsto\left(u^{2}: v^{2}: u v: v w: u w\right) .
$$

The map blows up the point $(0: 0: 1)$. The image is a degree 3 ruled surface. $V_{3}$ lies on a 3 -dimensional family of quadrics best described as the $2 \times 2$ subdeterminants of the matrix

$$
\left(\begin{array}{lll}
x_{0} & x_{2} & x_{4} \\
x_{2} & x_{1} & x_{3}
\end{array}\right) .
$$


42 (Quadric 3-folds). We have seen on our list in (41) that a smooth quadric 3fold can not contain a degree 3 or 4 projection of a Veronese surface. Thus for all non-degenerate maps the image is a quadric surface contained in $Q^{3}$.

Maps whose image is a smooth $Q^{2} \subset Q^{3}$ were described in (40,3) and

$$
\operatorname{Map}_{U P}\left(\mathbb{P}^{2}, Q^{3}\right) \stackrel{\text { bir }}{\sim} Q^{3} \times \mathbb{P}^{11} .
$$

This family has members over $k$ whenever $Q^{3}(k) \neq \emptyset$ and then $\operatorname{Map}_{U P}\left(\mathbb{P}^{2}, Q^{3}\right) \stackrel{\text { bir }}{\sim}$ $\mathbb{P}^{14}$.

Maps to a quadric cone $Q^{2,0} \subset Q^{3}$ form another family of dimension $3+11=14$. By $[38,1)$ they form a family

$$
\operatorname{Map}_{Q C}\left(\mathbb{P}^{2}, Q^{3}\right) \stackrel{\text { bir }}{\sim} \operatorname{OG}\left(\mathbb{P}^{1}, Q^{3}\right) \times \mathbb{P}^{11} .
$$

The second family has members over $k$ whenever $Q^{3}$ contains a $k$-line. This happens only if

$$
q\left(x_{0}, \ldots, x_{4}\right) \sim y_{0}^{2}+y_{1} y_{2}+y_{3} y_{4} .
$$

Both of these families have dimension 14, so we conclude that

$$
\operatorname{Map}_{2}\left(\mathbb{P}^{2}, Q^{3}\right) \stackrel{\text { bir }}{\sim}\left(Q^{3} \times \mathbb{P}^{11}\right) \amalg\left(\mathrm{OG}\left(\mathbb{P}^{1}, Q^{3}\right) \times \mathbb{P}^{11}\right) .
$$

43 (Projected Veronese surfaces). Let $V_{2} \subset Q^{n}$ be a projected Veronese surface with double line $\ell \subset V_{2}$.

The linear span $\left\langle V_{2}\right\rangle$ of $V_{2}$ has dimension 4 and $V_{2}$ lies in the 3-dimensional quadric $Q^{n} \cap\left\langle V_{2}\right\rangle$. As we noted in (42), $Q^{n} \cap\left\langle V_{2}\right\rangle$ has a unique singular point $p$. Thus $V_{2}$ determines a singular subquadric $Q^{3,0} \subset Q^{n}$ plus a line $\ell \subset Q^{3,0}$ through the vertex $p$ of $Q^{3,0}$. We can specify these data by first picking the line $\ell$, then the point $p \in \ell$ and finally a 3 -dimensional linear subspace $[\ell] \in L^{3} \subset \mathbb{P}\left(T_{p} Q^{n}\right)$.

Fix $Q^{3,0}:=\left(x_{1} x_{2}=x_{3} x_{4}\right) \subset \mathbb{P}^{4}$ and the line $\ell=\left(x_{2}=x_{3}=x_{4}=0\right)$. One easily computes that quadric sections of $Q^{3,0}$ that vanish doubly along $\ell$ are all of the form $x_{2} L\left(x_{0}, \ldots, x_{4}\right)+a_{3} x_{3}^{2}+a_{4} x_{4}^{2}$, thus their linear system has dimension 6 .

Putting these together we obtain that the Chow variety of projected Veronese surfaces is

$$
\begin{aligned}
\left.\operatorname{Chow}_{P V}\left(Q^{n}\right) \underset{\stackrel{\text { bir }}{\sim}}{\stackrel{\text { bir }}{\sim}} \operatorname{OG}\left(\mathbb{P}^{1}, Q^{n}\right) \times \mathbb{P}^{1} \times \mathbb{P}^{1}, Q^{n}\right) \times \mathbb{P}^{3 n-5} .
\end{aligned}
$$

Since the quadratic maps $\mathbb{P}^{2} \rightarrow V_{2}$ are birational, we conclude that

$$
\operatorname{Map}_{P V}\left(\mathbb{P}^{2}, Q^{n}\right) \stackrel{\text { bir }}{\sim} \mathrm{OG}\left(\mathbb{P}^{1}, Q^{n}\right) \times \mathbb{P}^{3 n+3} .
$$

\section{VERONESE SURFACES IN QUADRIC 4-FOLDS}

In the previous section we have enumerated all degenerate maps of $\mathbb{P}^{2}$ to a smooth quadric 4-fold. Among those, $\operatorname{Map}_{P V}\left(\mathbb{P}^{2}, Q^{4}\right)$ and $\operatorname{Map}_{Q P}\left(\mathbb{P}^{2}, Q^{4}\right)$ have dimension 20 and the others have dimension $<20$. Since the expected dimension of $\operatorname{Map}_{2}\left(\mathbb{P}^{2}, Q^{4}\right)$ is $6 \cdot 4-4=20$ by (36), once we prove that $\operatorname{dim} \operatorname{Map}_{V}\left(\mathbb{P}^{2}, Q^{4}\right)=20$, we will know that all the geometric irreducible components of $\operatorname{Map}_{2}\left(\mathbb{P}^{2}, Q^{4}\right)$ are given by $\operatorname{Map}_{V}\left(\mathbb{P}^{2}, Q^{4}\right), \operatorname{Map}_{P V}\left(\mathbb{P}^{2}, Q^{4}\right)$ and $\operatorname{Map}_{Q P}\left(\mathbb{P}^{2}, Q^{4}\right)$.

Thus it remains to describe the moduli space of Veronese surfaces in quadric 4 -folds. 
44 (Homology class of Veronese surfaces). The Chern class of $Q^{4}$ is $\left(1+H_{5}\right)^{6}(1+$ $\left.2 H_{5}\right)^{-1}$ where $H_{5}$ is the hyperplane class on $\mathbb{P}^{5}$. Restricting to $V$ this gives $(1+$ $\left.2 \mathrm{H}_{2}\right)^{6}\left(1+4 \mathrm{H}_{2}\right)^{-1}$ where $\mathrm{H}_{2}$ is the hyperplane class on $\mathbb{P}^{2}$ (note that the restriction of $H_{5}$ equals $\left.2 H_{2}\right)$. The Chern class of $\mathbb{P}^{2}$ is $\left(1+H_{2}\right)^{3}$, hence the Chern class of the normal bundle of $V \subset Q^{4}$ is

$$
c\left(N_{V, Q}\right)=\left(1+2 H_{2}\right)^{6}\left(1+4 H_{2}\right)^{-1}\left(1+H_{2}\right)^{-3}=1+5 H_{2}+10 H_{2}^{2} .
$$

Thus $(V \cdot V)=10$. Since $\left(V \cdot H_{5}^{2}\right)=4$ this leads to the possibilities $[V]=3[A]+[B]$ or $[V]=[A]+3[B]$, as computed in Ber1907, Sec.XV.13], Ber24, Sec.XVI.13]. This suggests, and we will see, that there are 2 distinct Veronese families.

We start with a duality between the 2 families of Veronese surfaces. Then we work with a special case when one side is a very singular specialization of Veronese surfaces to get our moduli description.

45 (Linking the two Veronese families). Let $Q^{4}$ be a smooth quadric 4 -fold and $V \subset Q^{4}$ a Veronese surface. $V$ is contained in a 5-dimensional family of quadrics in $\mathbb{P}^{5}$, thus in a 4-dimensional family of quadric sections of $Q^{4}$. Let $\left\langle Q_{1}, Q_{2}\right\rangle \subset$ $\left|\mathcal{O}_{Q^{4}}(2)(-V)\right|$ be any pencil. Then $Q^{4} \cap Q_{1} \cap Q_{2}$ is a degree 8 surface containing $V$. Thus we get a residual intersection

$$
Q^{4} \cap Q_{1} \cap Q_{2}=V \cup V^{\prime}
$$

where $V^{\prime}$ is another degree 4 surface contained in $Q^{4}$. If $[V]=3[A]+[B]$ then $\left[V^{\prime}\right]=[A]+3[B]$, so the correspondence $V \leftrightarrow V^{\prime}$ interchanges the 2 families. Most likely, a given $V$ will give all others by repeating such linkages but I have no proof of this.

Let us see some nice examples, using the representation of $V$ in the space of symmetric matrices. Our first pair is

$$
V:=\left(\begin{array}{ccc}
u^{2} & u v & u w \\
u v & v^{2} & v w \\
u w & v w & w^{2}
\end{array}\right) \text { and } \quad V^{\prime}:=\left(\begin{array}{ccc}
u^{2} & -u v & -u w \\
-u v & v^{2} & -v w \\
-u w & -v w & w^{2}
\end{array}\right) .
$$

Their union is defined by the symmetric subdeterminant equations

$$
x_{01}^{2}-x_{00} x_{11}=x_{02}^{2}-x_{00} x_{22}=x_{12}^{2}-x_{11} x_{22}=0 .
$$

The sum of the 3 equations gives

$$
Q^{4}:=\left(x_{01}^{2}+x_{02}^{2}+x_{12}^{2}-x_{00} x_{11}-x_{00} x_{22}-x_{11} x_{22}=0\right) ;
$$

it is an ellipsoid over $\mathbb{R}$; see $(23,4-5)$.

We can also take the 3 non-symmetric subdeterminant equations

$$
x_{01} x_{12}-x_{02} x_{11}=x_{12} x_{02}-x_{01} x_{22}=x_{01} x_{22}-x_{12} x_{02}=0 .
$$

In this case $V^{\prime \prime}$ is the union of four 2-planes, consisting of all matrices of the form

$$
\left(\begin{array}{lll}
* & 0 & 0 \\
0 & * & 0 \\
0 & 0 & *
\end{array}\right),\left(\begin{array}{lll}
* & * & 0 \\
* & * & 0 \\
0 & 0 & 0
\end{array}\right),\left(\begin{array}{lll}
0 & 0 & 0 \\
0 & * & * \\
0 & * & *
\end{array}\right) \text { and }\left(\begin{array}{ccc}
* & 0 & * \\
0 & 0 & 0 \\
* & 0 & *
\end{array}\right) \text {. }
$$

Note that the canonical class of the intersection of 3 quadrics in $\mathbb{P}^{5}$ is trivial, so $V \cup V^{\prime}$ is a singular K3 surface. Another way to construct these $V \cup V^{\prime}$ is the following.

A degree 6 elliptic normal curve $\tau: E \hookrightarrow \mathbb{P}^{5}$ is contained in 4 different Veronese surfaces, an observation going back to Coble Cob22. Indeed, for a given elliptic 
curve $E$ its degree 6 embeddings $\tau_{6}: E \hookrightarrow \mathbb{P}^{5}$ correspond to degree 6 line bundles $L_{6}:=\tau_{6}^{*} \mathcal{O}_{\mathbb{P}^{5}}(1)$ and degree 3 embeddings $\tau_{3}: E \hookrightarrow \mathbb{P}^{2}$ correspond to degree 3 line bundles $L_{3}:=\tau_{3}^{*} \mathcal{O}_{\mathbb{P}^{2}}(1)$. Combining $\tau_{3}$ with the Veronese embedding gives a degree 6 elliptic normal curve such that $L_{6}=L_{3}^{\otimes 2}$. Given $L_{6}$, there are 4 different $L_{3}$ satisfying this equation. These give the 4 different Veronese surfaces containing $\tau_{6}(E)$. The union of any 2 of them is a singular K3 surface.

Russo pointed out that the various degenerations of Veronsese surfaces can best be seen using the dual picture to be discussed in (48).

Next we study a special case when $V^{\prime}$ is singular, even reducible, but slightly better than the ones exhibited in $(45,5-6)$.

46 (A degenerate version). Let $p \in V \subset Q^{4} \subset \mathbb{P}^{5}$ be a pointed Veronese surface. Instead of working with all quadrics that contain $V$, we consider only quadric cones with vertex $p$ that contain $V$. These form a linear system $\left|2 H_{5}\right|(-V-2 p)$ of dimension 2. We claim that if $\left\langle Q_{1}, Q_{2}\right\rangle \subset\left|2 H_{5}\right|(-V-2 p)$ is a general pencil then

$$
Q^{4} \cap Q_{1} \cap Q_{2}=V \cup P_{1} \cup P_{2} \cup Q^{2},
$$

where the $P_{i}$ are 2-planes meeting at $p$ and $Q^{2}$ is a smooth quadric surface through $p$. This is easier to see by projecting everything from $p$. So we have $\pi: \mathbb{P}^{5} \rightarrow \mathbb{P}^{4}$ and $\pi(V)$ is a degree 3 surface $F_{3} \cong \mathbb{F}_{1}$, as discussed in (41). Thus $\left|2 H_{4}\right|\left(-F_{3}\right)$ has dimension 2 and if $\left\langle Q_{1}^{\prime}, Q_{2}^{\prime}\right\rangle \subset\left|2 H_{4}\right|\left(-F_{3}\right)$ is a general pencil then

$$
Q_{1}^{\prime} \cap Q_{2}^{\prime}=F_{3} \cup L^{2},
$$

where $L^{2}$ is a 2-plane that meets $F_{3}$ in a conic. This $L^{2}$ is the projection of the $Q^{2}$.

The planes $P_{i}$ are less visible after projection; we obtain them as follows. Write $(p \in V)$ as the image of $\phi:\left(p_{0}, \mathbb{P}^{2}\right) \hookrightarrow \mathbb{P}^{5}$. Note that $\phi^{-1}\left(V \cap T_{p} Q^{4}\right)$ is a degree 2 curve in $\mathbb{P}^{2}$ that is singular at $p_{0}$. Thus $\phi^{-1}\left(V \cap T_{p} Q^{4}\right)$ is a pair of lines in $\mathbb{P}^{2}$ that meet at $p_{0}$ and $V \cap T_{p} Q^{4}$ is a pair of conics $C_{i}$ meeting at $p$. Each $C_{i}$ spans a 2-plane $P_{i}$ which is contained every member of $\left|2 H_{\mathbb{P}^{5}}\right|(-V-2 p)$. Thus the $P_{i}$ do not depend on $\left\langle Q_{1}, Q_{2}\right\rangle$ but $Q^{2}$ does.

Note that the $\pi$-images of the $P_{i}$ are lines $L_{i} \subset F_{3}$ and $L^{2}$ meets both of them. Thus $P_{1} \cup P_{2} \cup Q^{2}$ is a surface where all 3 components meet at $p, P_{i} \cap Q^{2}$ are lines and $P_{i} \cap P_{2}=\{p\}$. These data specify a unique isomorphism class of surfaces in $\mathbb{P}^{5}$, up to $\operatorname{Aut}\left(\mathbb{P}^{5}\right)$.

This construction can be reversed. That is, assume that we have $P_{1} \cup P_{2} \cup Q^{2} \subset$ $Q^{4}$. Let $\left|2 H_{\mathbb{P}^{5}}\right|\left(-Q^{2}-P_{1}-P_{2}-2 p\right)$ denote that linear system of quadric cones with vertex $p$ that contain $P_{1} \cup P_{2} \cup Q^{2}$. We see that it has dimension 2. (This is best seen after projecting from $p$. Then we have the linear system of quadrics that contain a 2-plane $L^{2}$ and two lines $L_{i}$ that meet it.)

If $\left\langle Q_{1}, Q_{2}\right\rangle \subset\left|2 H_{5}\right|\left(-Q^{2}-P_{1}-P_{2}-2 p\right)$ is a general pencil then

$$
Q^{4} \cap Q_{1} \cap Q_{2}=V \cup P_{1} \cup P_{2} \cup Q^{2},
$$

where $V$ is a Veronese surface passing through $p$.

Let us next study the induced correspondence

$$
\{V\} \leftarrow\left\{V \cup Q^{2} \cup P_{1} \cup P_{2}\right\} \rightarrow\left\{P_{1} \cup P_{2}\right\} .
$$

We start with $[V] \in \operatorname{Chow}_{V}\left(Q^{4}\right)$. As we noted before Corollary 7 , the universal family over $\operatorname{Chow}_{V}\left(Q^{4}\right)$ is birationally trivial, thus pointed Veronese surfaces are birationally parametrized by $\operatorname{Chow}_{V}\left(Q^{4}\right) \times \mathbb{P}^{2}$. The choice of the pencil $\left\langle Q_{1}, Q_{2}\right\rangle \subset$ 
$\left|2 H_{\mathbb{P}^{5}}\right|(-V-2 p)$ is another $\mathbb{P}^{2}$-factor. Thus the left hand side of [46, 4$)$ is birational to

$$
\operatorname{Chow}_{V}\left(Q^{4}\right) \leftarrow \operatorname{Chow}_{V}\left(Q^{4}\right) \times \mathbb{P}^{4} \text {. }
$$

On the right hand side of (46,4) we start by choosing $P_{1} \cup P_{2}$. This is the same as picking $P_{1}, P_{2} \subset Q^{4}$ that are in the same geometric irreducible component of $\mathrm{OG}\left(\mathbb{P}^{2}, Q^{4}\right)$. This will be denoted by $\operatorname{Sym}_{a}^{2}\left(\mathrm{OG}\left(\mathbb{P}^{2}, Q^{4}\right)\right)$ in (69). Then we choose $Q^{2}$. As we noted above, this is equivalent to having 2 lines $L_{1}, L_{2} \subset \mathbb{P}^{4}$ and choosing a 2-plane $L^{2}$ that meet both of them. After fixing an auxiliary $\mathbb{P}_{2}^{2} \subset \mathbb{P}^{4}$, these are parametrized by $L_{1} \times L_{2} \times \mathbb{P}^{2}$. Note, however, that for us the $L_{1}, L_{2}$ are conjugate, thus instead of $L_{1} \times L_{2}$ we pick another auxiliary $\mathbb{P}_{0}^{2} \subset\left\langle L_{1}, L_{2}\right\rangle \cong \mathbb{P}^{3}$ and represent a point pair $\left\{p_{1}, p_{2}: p_{i} \in L_{i}\right\}$ by the intersection point $\left\langle p_{1}, p_{2}\right\rangle \cap \mathbb{P}_{0}^{2}$. (This is just a birational construction of the Weil restriction $\Re\left(L_{1}\right)$.) As before, the choice of the pencil $\left\langle Q_{1}, Q_{2}\right\rangle \subset\left|2 H_{5}\right|\left(-Q^{2}-P_{1}-P_{2}-2 p\right)$ is a $\operatorname{Grass}\left(\mathbb{P}^{1}, \mathbb{P}^{4}\right)$-factor. Thus the diagram (46, 4) is birational to

$$
\begin{array}{ccc}
\operatorname{Chow}_{V}\left(Q^{4}\right) \times \mathbb{P}^{4} & \stackrel{\text { bir }}{\sim} & \operatorname{Sym}_{a}^{2}\left(\mathrm{OG}\left(\mathbb{P}^{2}, Q^{4}\right)\right) \times \mathbb{P}^{10} \\
\downarrow & & \\
\operatorname{Chow}_{V}\left(Q^{4}\right) & & \operatorname{Sym}_{a}^{2}\left(\mathrm{OG}^{2}\left(\mathbb{P}^{2}, Q^{4}\right)\right) .
\end{array}
$$

We finally use 669,4$)$ to get that

$$
\operatorname{Chow}_{V}\left(Q^{4}\right) \times \mathbb{P}^{4} \stackrel{\text { bir }}{\sim}\left(t^{2}+\Delta=0\right) \times Q^{4} \times \mathbb{P}^{12} .
$$

Remark 47. Another family of degree 4 surfaces in $Q^{4}$ can be obtained starting with 2 disjoint planes $P_{1}, P_{2} \subset Q^{4}$. A general member of this family is $\mathbb{P}^{1} \times \mathbb{P}^{1}$ embedded by $\mathcal{O}_{\mathbb{P}^{1} \times \mathbb{P}^{1}}(2,1)$. It is better to view such a surface as the product of a conic with $\mathbb{P}^{1}$, thus we have

$$
C \times \mathbb{P}^{1} \subset \mathbb{P}^{2} \times \mathbb{P}^{1} \stackrel{j}{\hookrightarrow} \mathbb{P}^{5},
$$

where $j$ is the embedding given by $\mathcal{O}_{\mathbb{P}^{2} \times \mathbb{P}^{1}}(1,1)$. The residual intersection of $Q^{4}$ with $j\left(\mathbb{P}^{2} \times \mathbb{P}^{1}\right)$ is a pair of planes $\mathbb{P}^{2} \times\left\{p_{1}, p_{2}\right\}$. Conversely, given a disjoint pair of conjugate planes $P_{1}, P_{2} \subset Q^{4}$ and an isomorphism $\tau: P_{1} \cong P_{2}$, we get an embbeding $P_{1} \amalg P_{2} \subset j\left(\mathbb{P}^{2} \times \mathbb{P}^{1}\right)$. The residual intersection of $j\left(\mathbb{P}^{2} \times \mathbb{P}^{1}\right)$ with $Q^{4}$ gives a required degree 4 surface in $Q^{4}$. This shows that this irreducible component of $\operatorname{Chow}\left(Q^{4}\right)$ is birational to $\operatorname{Sym}_{s}^{2}\left(\mathrm{OG}\left(\mathbb{P}^{2}, Q^{4}\right)\right) \times \mathbb{P}^{8}$ where $\operatorname{Sym}_{s}^{2}\left(\mathrm{OG}\left(\mathbb{P}^{2}, Q^{4}\right)\right)$ is the irreducible component of $\operatorname{Sym}^{2}\left(\mathrm{OG}\left(\mathbb{P}^{2}, Q^{4}\right)\right)$ parametrizing disjoint pairs of 2-panes; see (69) for details.

\section{Quadratic maps up to Coordinate changes}

Here we study $\operatorname{Map}_{2}\left(\mathbb{P}^{2}, Q^{4}\right)$, up to coordinate changes by $\operatorname{Aut}\left(\mathbb{P}^{2}\right) \times \operatorname{Aut}\left(Q^{4}\right)$. The 3 types of irreducible components of $\operatorname{Map}_{2}\left(\mathbb{P}^{2}, Q^{4}\right)$ behave differently. There is especially nice geometry behind the Veronese maps. The following classical facts can be found in [Ber1907, Cap.XV], Ber24, Kap.XVI], [SR49, p.188] or [ESB89.

48 (Equations of Veronese surfaces). The equations of the Veronese surface are especially clear if we think of the ambient $\mathbb{P}^{5}$ as the projectivization of the space of $3 \times 3$ symmetric matrices. Thus we consider $\mathbb{P}_{\mathbf{u}}^{2}$ with coordinates $\left\{u_{0}, u_{1}, u_{2}\right\}$ and

$\mathbb{P}_{\mathbf{x}}^{5}$ with coordinates $\left\{x_{i j}\right\}$ for $0 \leq i \leq j \leq 2$. The Veronese surface $V=V_{\mathbf{x}} \subset \mathbb{P}_{\mathbf{x}}^{5}$ is the image of the map $\phi: \mathbb{P}_{\mathbf{u}}^{2} \hookrightarrow \mathbb{P}_{\mathbf{x}}^{5}$ given by

$$
x_{i j}=u_{i} u_{j} \text { for } \quad 0 \leq i \leq j \leq 2 .
$$


In order to describe the equations defining $V$, consider the matrix

$$
M:=\left(\begin{array}{lll}
x_{00} & x_{01} & x_{02} \\
x_{01} & x_{11} & x_{12} \\
x_{02} & x_{12} & x_{22}
\end{array}\right) .
$$

$V$ is defined by the equations

$$
\operatorname{rank} M \leq 1, \quad \text { equivalently, } x_{i j} x_{k \ell}=x_{i \ell} x_{k j},
$$

(where we set $x_{i j}=x_{j i}$ ) and the secant variety of $V$ is given by $\operatorname{det} M=0$. The quadrics containing $V$ are given by (linear combinations of) the $2 \times 2$ subdeterminants of $M$. These define a birational map

$$
\sigma_{\mathbf{x}}: \mathbb{P}_{\mathbf{x}}^{5} \rightarrow \mathbb{P}_{\mathbf{y}}^{5} \quad \text { given by } \quad y_{i j}=(-1)^{i+j} M_{j i} .
$$

Geometrically, we obtain $\sigma_{\mathbf{x}}$ by blowing up $V_{\mathbf{x}} \subset \mathbb{P}_{\mathbf{x}}^{5}$ and then contracting the (birational transform of the) secant variety of $V_{\mathbf{x}} \subset \mathbb{P}_{\mathbf{x}}^{5}$. Note that $\sigma_{\mathbf{x}}: \mathbb{P}_{\mathbf{x}}^{5} \rightarrow \mathbb{P}_{\mathbf{y}}^{5}$ and $\sigma_{\mathbf{y}}: \mathbb{P}_{\mathbf{y}}^{5} \rightarrow \mathbb{P}_{\mathbf{x}}^{5}$ are inverses of each other since, for an $r \times r$ matrix,

$$
\operatorname{adj}(\operatorname{adj}(M))=(\operatorname{det} M)^{r-2} \cdot M \text {. }
$$

(For this reason, one usually views $\sigma:=\sigma_{\mathbf{x}}$ as an involution of $\mathbb{P}^{5}$, but for us it will be convenient to distinguish the source $\mathbb{P}^{5}$ from the target $\mathbb{P}^{5}$.) Note that $\sigma_{\mathbf{x}}$ maps quadrics that contain $V$ to hyperplanes; this correspondence is given as follows. Restricting the above factorization of $\sigma_{\mathbf{x}}$ to a quadric $Q^{4}$ we obtain the following. First we blow up $V_{\mathbf{x}} \subset Q^{4}$ and then contract the (birational transform of the) secant variety of $V_{\mathbf{x}}$ in $Q^{4}$; that is, the union of those secant lines of $V_{\mathbf{x}}$ that are contained in $Q^{4}$. For the inverse, we blow up $V_{\mathbf{y}} \cap H^{4}$ and then contract the (birational transform of the) secant variety of $V_{\mathbf{y}} \cap H^{4}$. If $Q^{4}$ is smooth then the blow-up of $V_{\mathbf{x}}$ is smooth but the blow-up of $V_{\mathbf{y}} \cap H^{4}$ is smooth iff $V_{\mathbf{y}} \cap H^{4}$ is smooth. Thus we get the following.

Claim 48.6. $\sigma_{\mathbf{x}}$ establishes a one-to-one correspondence between

(a) pairs $V_{\mathbf{x}} \subset Q^{4}$, up to isomorphism and

(b) pairs $V_{\mathbf{y}} \cap H^{4} \subset H^{4}$, up to isomorphism.

Furthermore, $Q^{4}$ is smooth iff the corresponding $V_{\mathbf{y}} \cap H^{4}$ is smooth.

I have not been able to track down the original author of this claim. For the geometric version all the ingredients are in [Ber1907, Secs.XV.9-10], Cob22, Thm.17] and it is mentioned as an exercise in [SR49, p.188].

Next observe that $V_{\mathbf{y}} \cap H^{4}$ is a degree 4 geometrically rational curve in $\mathbb{P}^{4}$, thus it is obtained as the image of a plane conic $C$ embedded by $\left|-2 K_{C}\right|$. In particular, the pair $V_{\mathbf{y}} \cap H^{4} \subset H^{4}$ is uniquely determined by $V_{\mathbf{y}} \cap H^{4}$, up to isomorphism. This proves Complement 9 .

One can write down the correspondence between conics and Veronese surfaces contained in a smooth quadric as follows.

49 (Explicit formulas). Any plane conic can be given by a diagonal equation

$$
C:=\left(a v_{0}^{2}+b v_{1}^{2}+c v_{2}^{2}=0\right) \subset \mathbb{P}_{\mathbf{v}}^{2} .
$$

This conic is cut out of the Veronese surface $\mathbb{P}_{\mathbf{v}}^{2} \hookrightarrow \mathbb{P}_{\mathbf{y}}^{5}$, by the hyperplane

$$
H_{C}^{4}:=\left(a y_{00}+b y_{11}+c y_{22}=0\right) \subset \mathbb{P}_{\mathbf{y}}^{5},
$$


and taking its preimage by $\sigma_{\mathbf{x}}$ gives the quadric

$$
\left.Q_{C}^{4}:=\left(a\left(x_{11} x_{22}-x_{12}^{2}\right)+b\left(x_{00} x_{22}-x_{02}^{2}\right)+c\left(x_{00} x_{11}-x_{01}^{2}\right)=0\right) \subset \mathbb{P}_{\mathbf{x}}^{5} . \quad \text { [49, } 3\right)
$$

We can assume that $c=1$ and rearrange the equation into

$$
\left(x_{00}+a x_{22}\right)\left(x_{11}+b x_{22}\right)=x_{01}^{2}+a x_{12}^{2}+b x_{02}^{2}+a b x_{22}^{2} .
$$

Thus we see that $Q_{C}^{4}$ has a $k$-point and its Witt reduction is the quadric surface

$$
Q_{C}^{2}:=\left(x_{01}^{2}+a x_{12}^{2}+b x_{02}^{2}+a b x_{22}^{2}=0\right) \cong C \times C,
$$

where the isomorphism holds by Paragraph 50. This shows that the conic $C$ and the quadric $Q_{C}^{4}$ uniquely determine each other, up to isomorphism.

We can also use the the symmetric subdeterminant equations (45,3) to get the quadric

$$
Q_{a, b}^{4}:=a\left(x_{01}^{2}-x_{00} x_{11}\right)+b\left(x_{02}^{2}-x_{00} x_{22}\right)+a b\left(x_{12}^{2}-x_{11} x_{22}\right)=0 .
$$

The equation can be rewritten in the usual form as

$$
x_{00}^{2}+a x_{01}^{2}+b x_{02}^{2}+a b x_{12}^{2}=\left(x_{00}+a x_{11}\right)\left(x_{00}+b x_{22}\right) .
$$

50 (Decomposable quadric surfaces). Let $C:=\left(a x_{0}^{2}+b x_{1}^{2}+c x_{2}^{2}=0\right)$. Then

$$
\begin{aligned}
\psi: & \left(x_{0}: x_{1}: x_{2}\right) \times\left(y_{0}: y_{1}: y_{2}\right) \\
& \mapsto\left(x_{1} y_{2}-x_{2} y_{1}:-x_{0} y_{2}+x_{2} y_{0}: x_{0} y_{1}-x_{1} y_{0}: a x_{0} y_{0}+b x_{1} y_{1}+c x_{2} y_{2}\right)
\end{aligned}
$$

is an isomorphism of $C \times C$ and the quadric

$$
Q:=\left(b c z_{0}^{2}+a c z_{1}^{2}+a b z_{2}^{2}+z_{3}^{2}=0\right) .
$$

Note that $\psi$ is given by 4 sections of $\mathcal{O}_{C \times C}(2,2)$ that vanish along the diagonal.

Explanation. The first 3 components of $\psi$ map 2 points on $C$ to the line connecting them. This gives a morphism to (the dual) $\mathbb{P}^{2}$ and its branch locus is the set of tangent lines of $C$. Tangent lines have coefficients $\left(2 a x_{0}, 2 b x_{1}, 2 c x_{2}\right)$ and these satisfy the equation $b c\left(2 a x_{0}\right)^{2}+a c\left(2 b x_{1}\right)^{2}+a b\left(2 c x_{2}\right)^{2}=0$. This shows that $b c z_{0}^{2}+a c z_{1}^{2}+a b z_{2}^{2}$ is (a constant times) a square.

The inverse of $\psi$ is given by

$$
\begin{aligned}
& \left(x_{0}: x_{1}: x_{2}\right)=\left(z_{1} z_{3}-b z_{0} z_{2}:-z_{0} z_{3}-a z_{1} z_{2}: b z_{0}^{2}+a z_{1}^{2}\right) \text { and } \\
& \left(y_{0}: y_{1}: y_{2}\right)=\left(z_{1} z_{3}+b z_{0} z_{2}:+z_{0} z_{3}-a z_{1} z_{2}: b z_{0}^{2}+a z_{1}^{2}\right) .
\end{aligned}
$$

Next we prove the other claims about maps up to coordinate changes.

51 (Proof of Proposition 12). If $Q^{4} \subset \mathbb{P}^{5}$ contains a plane, we may assume that it is $\left(x_{3}=x_{4}=x_{5}=0\right)$. Then $\mathbb{P}^{2} \rightarrow Q^{4}$ is of the form

$$
(u: v: w) \mapsto\left(q_{0}(u, v, w), q_{1}(u, v, w), q_{2}(u, v, w), 0,0,0\right) .
$$

where the $q_{i}$ are homogeneous of degree 2. The $q_{i}$ span a base point free net of conics and, if char $k \neq 3$ then any such net can be written as

$$
\left\langle\frac{\partial C(u, v, w)}{\partial u}, \frac{\partial C(u, v, w)}{\partial v}, \frac{\partial C(u, v, w)}{\partial w}\right\rangle,
$$

where $C(u, v, w)$ is a homogeneous cubic; see [SR49, p.42].

Thus the equation of $Q^{4}$ can be written as $x_{0} \ell_{3}+x_{1} \ell_{4}+x_{2} \ell_{5}=0$ where the $\ell_{i}$ are linear. Then $Q$ is smooth iff the 6 linear terms in the equation are linearly independent, hence we can change $x_{3}, x_{4}, x_{5}$ to $x_{i}:=\ell_{i}$ for $i=3,4,5$. 
This gives an isomorphism between the moduli space of quadruple planes (up to $\operatorname{Aut}\left(\mathbb{P}^{2}\right) \times \operatorname{Aut}\left(Q^{4}\right)$ ) and the moduli space of plane cubics (up to $\operatorname{Aut}\left(\mathbb{P}^{2}\right)$ ). This completes the proof of Proposition 12 .

Note that we have in fact proved the much more precise statement: there is an isomorphism of stacks

$$
\operatorname{Aut}\left(\mathbb{P}^{2}\right) \backslash \operatorname{Map}_{Q P}^{\circ}\left(\mathbb{P}^{2}, Q^{4}\right) / \operatorname{Aut}\left(Q^{4}\right) \cong \operatorname{Div}_{3}^{\circ}\left(\mathbb{P}^{2}\right) / \operatorname{Aut}\left(\mathbb{P}^{2}\right)
$$

where $\operatorname{Div}_{3}^{\circ}\left(\mathbb{P}^{2}\right)$ parametrizes smooth, degree 3 plane curves. Equivalently, for any field $k$ we have an equality of sets

$$
\mathrm{PGL}_{3}(k) \backslash \operatorname{Map}_{Q P}^{\circ}\left(\mathbb{P}^{2}, Q^{4}\right)(k) / \mathrm{O}\left(Q^{4}, k\right) \cong \operatorname{Div}_{3}^{\circ}\left(\mathbb{P}^{2}\right)(k) / \mathrm{PGL}_{3}(k) .
$$

52 (Proof of Proposition 11). Let $S \subset Q^{4}$ be a projected Veronese surface with singular line $L \subset S$. Then $S$ is contained in a unique $Q^{3,0} \subset Q^{4}$ and projecting $S$ from the vertex of $Q^{3,0}$ gives a birational map $\pi: S \rightarrow Q^{2}$. The image of the singular line in $S$ is a point $p \in Q^{2}$.

Conversely, given $p \in Q^{2}$ we first blow up $p$ to get $B_{p} Q^{2}$ with exceptional curve $E$ and then obtain $\tau: B_{p} Q^{2} \rightarrow S$ that is an isomorphism $B_{p} Q^{2} \backslash E \cong S \backslash L$ outside $E$ but whose restriction $\tau_{E}: E \rightarrow L$ has degree 2 . We can identify $\tau_{E}: E \rightarrow L$ with a degree 2 homogeneous polynomial on $T_{p} Q^{2}$, up to scalars.

Note that the quadric $Q^{2}$ itself gives a quadratic form on $T_{p} Q^{2}$; in suitable coordinates it is $x^{2}+\Delta y^{2}$ where $\Delta$ is the discriminant of $Q^{4}$. Thus, up to isomorphisms, $S$ is determined by $\operatorname{Sym}^{2}\left(\mathbb{P}^{1}\right) / \mathrm{O}\left(x^{2}+\Delta y^{2}\right)$.

53 (Proof of Proposition 18). Assume that $Q^{5}$ contains a Veronese surface $V$. The linear span of $V$ intersects $Q^{5}$ in a 4 -dimensional subquadric $Q_{V}^{4}$. As we noted in Paragraph 41, a Veronese surface is not contained in any 4-dimensional quadric with an isolated singularity, thus $Q_{V}^{4}$ is smooth.

Fix now an equation $q\left(x_{0}, \ldots, x_{6}\right)=0$ for $Q^{5}$. Using Proposition 11 after a coordinate change we can write $q$ as

$$
A\left(x_{0} x_{5}+x_{1}^{2}+a x_{2}^{2}+b x_{3}^{2}+a b x_{4}^{2}\right)+C x_{6}^{2}
$$

where $x_{6}=0$ defines $Q_{V}^{4}$. Note that the discriminant of the form in parenthesis is -1 , thus $-C / \Delta(q)$ is a square. We can thus choose $C=-\Delta(q)$, independent of the original choice of $V \subset Q^{5}$. By Witt's cancellation theorem (21) this shows that $Q_{V}^{4}$ is uniquely determined by $Q^{5}$, up to isomorphism. Thus Proposition 11] shows that the pair $\left(V \subset Q^{5}\right)$ is unique up to isomorphism.

\section{QUADRICS OF DIMENSION $\geq 5$}

In Section 4 we proved that every irreducible component of $\operatorname{Map}_{2}\left(\mathbb{P}^{2}, Q^{n}\right)$ has dimension $\geq 6 n-4$ and we computed the spaces that parametrize degenerate maps. In all cases they have dimension $<6 n-4$ provided $n \geq 5$. Thus $\operatorname{Map}_{V}^{\circ}\left(\mathbb{P}^{2}, Q^{n}\right)$ is dense in $\operatorname{Map}_{2}\left(\mathbb{P}^{2}, Q^{n}\right)$ for every $n \geq 5$. In particular, projected Veronese surfaces and quadruple planes can be deformed to Veronese surfaces in a 5-dimensional quadric. We give some concrete examples of this in (55).

For now we focus on describing the birational type of $\operatorname{Map}_{V}^{\circ}\left(\mathbb{P}^{2}, Q^{n}\right)$.

54 (Proof of Theorem 13). We closely follow the arguments in (46). As in (46, 4), the key point is to study the correspondence

$$
\{V\} \leftarrow\left\{V \cup Q^{2} \cup P_{1} \cup P_{2} \subset Q^{4}\right\} \rightarrow\left\{P_{1} \cup P_{2}\right\} .
$$


Here $V$ is a Veronese surface in $Q^{n}, Q^{4} \subset Q^{n}$ is a smooth subquadric, $V \cup Q^{2} \cup P_{1} \cup P_{2}$ is a complete intersection of 3 quadrics in $\mathbb{P}^{n+1}$ and $V \cup Q^{2} \cup P_{1} \cup P_{2} \subset Q^{4}$ is the same configuration as in (46).

Since a Veronese surface spans a $\mathbb{P}^{5}$, it uniquely determines the 4-dimensional subquadric $Q^{4} \subset Q^{n}$ that contains it. Thus, as before, the left hand side of [54, 1 ) is birational to

$$
\operatorname{Chow}_{V}\left(Q^{n}\right) \leftarrow \operatorname{Chow}_{V}\left(Q^{n}\right) \times \mathbb{P}^{4} .
$$

On the right hand side of $(54,1)$ we start with the family of pairs of intersecting planes $\left\{P_{1} \cup P_{2}\right\}$. Let us denote this by $\operatorname{Sym}_{i}^{2}\left(\mathrm{OG}\left(\mathbb{P}^{2}, Q^{n}\right)\right)$. (This is a locally closed subset of $\operatorname{Sym}^{2}\left(\mathrm{OG}\left(\mathbb{P}^{2}, Q^{4}\right)\right)$, but, unlike in the $n=4$ case, it is not an irreducible component. However, it is a dense subset of an irreducible component of $\operatorname{Hilb}_{t^{2}+3 t+1}\left(Q^{n}\right)$, parametrizing subschemes with Hilbert polynomial $t^{2}+3 t+1$.)

Sending a pair $P_{1} \cup P_{2}$ to the intersection point gives a map

$$
\Pi: \operatorname{Sym}_{i}^{2}\left(\mathrm{OG}\left(\mathbb{P}^{2}, Q^{n}\right)\right) \rightarrow Q^{n}
$$

whose fiber over $p \in Q^{n}$ is a dense open subset of $\operatorname{Sym}^{2}\left(\mathrm{OG}\left(\mathbb{P}^{1}, Q_{p}^{n-2}\right)\right)$. In general the fibration given by $\Pi$ is not birationally trivial, but it is so if $Q^{n}(k) \neq \emptyset$; this follows from (20)3). We obtain that if $Q^{n}(k) \neq \emptyset$ then

$$
\operatorname{Sym}_{i}^{2}\left(\mathrm{OG}\left(\mathbb{P}^{2}, Q^{n}\right)\right) \stackrel{\text { bir }}{\sim} Q^{n} \times \operatorname{Sym}^{2}\left(\mathrm{OG}\left(\mathbb{P}^{1}, Q_{W}^{n-2}\right)\right) .
$$

The rest is very much like before, except that the linear span of $P_{1} \cup P_{2}$ is 4dimensional, thus the choice of $Q^{4} \supset P_{1} \cup P_{2}$ is equivalent to choosing a 5dimensional linear subspace containing $P_{1} \cup P_{2}$. So we get an extra factor of $\mathbb{P}^{n-4}$. At the end we obtain that the diagram (541) is birational to

$$
\begin{array}{ccc}
\operatorname{Chow}_{V}\left(Q^{n}\right) \times \mathbb{P}^{4} & \stackrel{\text { bir }}{\sim} & \operatorname{Sym}_{i}^{2}\left(\mathrm{OG}\left(\mathbb{P}^{2}, Q^{n}\right)\right) \times \mathbb{P}^{n-4} \times \mathbb{P}^{10} \\
\downarrow & \downarrow & \operatorname{Sym}_{i}^{2}\left(\mathrm{OG}^{10}\left(\mathbb{P}^{2}, Q^{n}\right)\right) .
\end{array}
$$

If $Q^{n}(k) \neq \emptyset$ then $(54,4)$ further gives that

$$
\operatorname{Chow}_{V}\left(Q^{n}\right) \times \mathbb{P}^{4} \stackrel{\text { bir }}{\sim} \operatorname{Sym}^{2}\left(\mathrm{OG}\left(\mathbb{P}^{1}, Q_{W}^{n-2}\right)\right) \times \mathbb{P}^{2 n+6} .
$$

Since $\operatorname{Map}_{V}^{\circ}\left(\mathbb{P}^{2}, Q^{n}\right) \stackrel{\text { bir }}{\sim} \operatorname{Chow}_{V}\left(Q^{4}\right) \times \mathbb{P}^{8}$, we see that (54,6) implies (13,2). As we already noted, 668,2$)$ shows that $(13,1)$ and $(13,2)$ are equivalent.

Example 55. We give examples of projected Veronese surfaces and quadruple planes deforming to Veronese surfaces in a 5 -dimensional quadric.

First consider the quadric $Q^{5}:=\left(x_{0} x_{1}+x_{2} x_{3}=x_{4}^{2}+a x_{5}^{2}+x_{6}^{2}\right)$. Pick any degree 2 polynomial $q_{1}(u, v)$ that is relatively prime to $u^{2}+a v^{2}$. Pick any degree 2 polynomial $q_{6}(u, v)$ and write $q_{6}^{2}=q_{0} q+q_{3}\left(u^{2}+a v^{2}\right)$ where $q_{0}, q_{3}$ have degree 2 . Consider the family of maps

$$
\phi_{t}:(u: v: w) \mapsto\left(t^{2} q_{0}: q_{1}: u^{2}+a v^{2}: w^{2}+t^{2} q_{3}: u w: v w: t q_{6}\right)
$$

Note that $\phi_{0}$ is a projected Veronese surface but the image of $\phi_{t}$ is a Veronese surface for $t \neq 0$.

Second, for the quadric $Q^{5}:=\left(x_{0} x_{3}+x_{1} x_{4}+x_{2} x_{5}=x_{6}^{2}\right)$ consider the family of maps $\phi_{t}: \mathbb{P}^{2} \rightarrow Q^{5}$ given by

$\phi_{t}:(u: v: w) \mapsto\left(u^{2}: v^{2}: w^{2}:(t v+t w)^{2}:(t u+t w)^{2}:(t u+t v)^{2}: t u v+t u w+t v w\right)$. 
Note that $\phi_{0}$ is a quadruple plane but the image of $\phi_{t}$ is a Veronese surface for $t \neq 0$.

\section{Orthogonal Grassmannians}

In the next two sections we discuss various facts about orthogonal Grassmannians that we used earlier. For the current applications we mainly need symmetric squares of $\mathrm{OG}\left(\mathbb{P}^{r}, Q^{n}\right)$ for $r=1,2$. We focus on these cases but give more general statements when possible.

Definition 56 (Orthogonal Grassmannians). The scheme parametrizing of all $m$ dimensional linear spaces contained in a smooth quadric $Q^{n}$ is called the orthogonal Grassmannian, denoted by $\mathrm{OG}\left(\mathbb{P}^{m}, Q^{n}\right)$.

Over an algebraically closed field the orthogonal Grassmannian is usually denoted by $\mathrm{OG}(m+1, n+2)$; the notation suggests $(m+1)$-dimensional $q$-isotropic sub-vector spaces of $k^{n+2}$ where $q$ is a non-degenerate quadratic form.

The orthogonal Grassmannian OG $(m+1, n+2)$ is a smooth projective variety that is homogeneous under the orthogonal group $\mathrm{O}(q)$.

The dimension of orthogonal Grassmannians can be computed several ways. For example, an easy application of (57/2) shows that the universal $\mathbb{P}^{m}$-bundle over $\mathrm{OG}(m+1, n+2)$ is an $\mathrm{OG}(m, n)$-bundle over $Q^{n}$. Thus $\operatorname{dim} \mathrm{OG}(m+1, n+2)=$

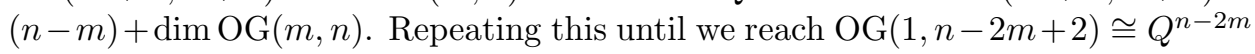
we get that

$$
\operatorname{dim} \operatorname{OG}(m+1, n+2)=\frac{1}{2}(2 n-3 m)(m+1) .
$$

This also shows that $\mathrm{OG}(m+1, n+2)$ is nonempty iff $2 m \leq n$, irreducible for $2 m<n$ and has 2 irreducible components for $2 m=n$; see (62). (Over an algebraically closed field some authors use OG( $m+1,2 m+2)$ to denote any one of these components.)

Thus OG( $\left(\mathbb{P}^{m}, Q^{n}\right)$ is a smooth projective $k$-variety that is homogeneous under the group $\operatorname{PGO}\left(Q^{n}\right)=\operatorname{Aut}\left(Q^{n}\right)$.

Intersection theory of orthogonal Grassmannians is studied in Cos14 and their quantum cohomology in KT04, BKT15. Here we aim to study the birational properties of orthogonal Grassmannians and their symmetric powers. Let us start with the existence of $k$-points.

57 (Basic correspondences). A basic tool comparing different orthogonal Grassmannians is the variety parametrizing pairs of linear spaces $L^{r} \subset L^{m}$ contained in $Q^{n}$. Let us denote it by $\mathrm{OF}\left(\mathbb{P}^{r}, \mathbb{P}^{m}, Q^{n}\right)$ (for orthogonal flags). Forgetting one of the linear spaces gives two morphisms

$$
\mathrm{OG}\left(\mathbb{P}^{r}, Q^{n}\right) \stackrel{\pi_{r}}{\longleftarrow} \mathrm{OF}\left(\mathbb{P}^{r}, \mathbb{P}^{m}, Q^{n}\right) \stackrel{\pi_{m}}{\longrightarrow} \mathrm{OG}\left(\mathbb{P}^{m}, Q^{n}\right) .
$$

The fiber of $\pi_{m}$ over $L^{m} \subset Q^{n}$ is the (ordinary) Grassmannian Grass $\left(\mathbb{P}^{r}, L^{m}\right)$.

We claim that the fiber of $\pi_{r}$ over $L:=L^{r} \subset Q^{n}$ is an orthogonal Grassmannian $\mathrm{OG}\left(\mathbb{P}^{m-r-1}, Q_{L}^{n-2 r-2}\right)$ that can be constructed as follows. First choose coordinates such that

$$
q\left(x_{0}, \ldots, x_{n+1}\right) \sim y_{0} y_{1}+\cdots+y_{2 r} y_{2 r+1}+q_{n-2 r}\left(y_{2 r+2}, \ldots, y_{n+1}\right)
$$

and $L^{r}=\left(y_{1}=y_{3}=\cdots=y_{2 r+1}=y_{2 r+2}=y_{2 r+3}=\cdots=y_{n+1}=0\right)$. Then we can identify an $L^{m}$ satisfying $L^{r} \subset L^{m} \subset Q^{n}$ with a linear space

$$
L^{m-r-1} \subset Q_{L}^{n-2 r-2}:=\left(q_{n-2 r}\left(y_{2 r+2}, \ldots, y_{n+1}\right)=0\right) .
$$


Geometrically one can obtain $Q_{L}^{n-2 r-2}$ as follows. First take

$$
Q_{L}^{n-r-1, r}:=\cap_{p \in L} T_{p} Q^{n} .
$$

It is a quadric of dimension $n-r-1$ that is singular along $L$. Then $Q_{L}^{n-r-1, r}$ is the cone over $Q_{L}^{n-2 r-2}$ with vertex $L$.

Two cases are especially useful. If $r=0$ then we get

$$
Q^{n} \stackrel{\pi_{0}}{\longleftarrow} \mathrm{OF}\left(\mathbb{P}^{0}, \mathbb{P}^{m}, Q^{n}\right) \stackrel{\pi_{m}}{\longrightarrow} \mathrm{OG}\left(\mathbb{P}^{m}, Q^{n}\right)
$$

where the fiber of $\pi_{m}$ over $L^{m} \subset Q^{n}$ is $L^{m}$ while the fiber of $\pi_{0}$ over $p \in Q^{n}$ is the orthogonal Grassmannian $\mathrm{OG}\left(\mathbb{P}^{m-1}, Q_{p}^{n-2}\right)$.

The other interesting case is $r=m-1$. Then we have

$$
\mathrm{OG}\left(\mathbb{P}^{m-1}, Q^{n}\right) \stackrel{\pi_{m-1}}{\longleftarrow} \mathrm{OF}\left(\mathbb{P}^{m-1}, \mathbb{P}^{m}, Q^{n}\right) \stackrel{\pi_{m}}{\longrightarrow} \mathrm{OG}\left(\mathbb{P}^{m}, Q^{n}\right) \text {. }
$$

Here $\pi_{m}$ is a $\mathbb{P}^{m}$-bundle (the dual of the natural $\mathbb{P}^{m}$-bundle) while the fiber of $\pi_{m-1}$ over $L^{m-1} \subset Q^{n}$ is the quadric $\left.Q_{L}^{n-2 m}\right)$.

Claim 57.4. Using the notation of (57,1),

(1) $\pi_{m}$ is a Zariski locally trivial $\mathbb{P}^{m}$-bundle and

(2) $\pi_{r}$ is a Zariski locally trivial $\mathrm{OG}\left(\mathbb{P}^{m-r-1}, Q_{M}^{n-2 r}\right)$-bundle if there is a linear subspace $M^{r} \subset Q^{n}$ defined over $k$.

Proof. If $r=0$ then $\pi_{m}$ is a $\mathbb{P}^{m}$-bundle and the pull-back of $\mathcal{O}_{\mathbb{P}^{n+1}}(1)$ gives a relative $\mathcal{O}(1)$. Thus the $\mathbb{P}^{m}$-bundle is Zariski locally trivial and so are its Grassmann bundles, proving the first part.

By contrast, $\pi_{r}$ is usually not Zariski locally trivial. However, assume that we have $M:=M^{r} \subset Q^{n}$ defined over $k$. As in (20]2), for any $L:=L^{r} \subset Q^{n} \backslash Q_{M}^{n-r-1, r}$ we get natural isomorphisms (defined over $k(L)$ )

$$
Q_{L}^{n-2 r-2} \cong Q_{L}^{n-r-1, r} \cap Q_{M}^{n-r-1, r} \cong Q_{M}^{n-2 r-2} .
$$

These trivialize the $Q_{L}^{n-2 r-2}$-bundle over the open set parametrizing those $L$ that are disjoint from $Q_{M}^{n-r-1, r}$. Thus the OG( $\left.\mathbb{P}^{m-r-1}, Q_{L}^{n-2 r-2}\right)$-bundle is also trivialized.

Proposition 58. Let $Q^{n}$ be a smooth quadric such that $Q^{n}(k) \neq \emptyset$. Then

$$
\mathrm{OG}\left(\mathbb{P}^{m}, Q^{n}\right) \stackrel{\text { bir }}{\sim} \mathrm{OG}\left(\mathbb{P}^{m-1}, Q_{W}^{n-2}\right) \times \mathbb{P}^{n-m} .
$$

Proof. Pick a $k$ point $p \in Q^{n}$ and consider the diagram (57,2)

$$
Q^{n} \stackrel{\pi_{0}}{\longleftarrow} \mathrm{OF}\left(\mathbb{P}^{0}, \mathbb{P}^{m}, Q^{n}\right) \stackrel{\pi_{m}}{\longrightarrow} \mathrm{OG}\left(\mathbb{P}^{m}, Q^{n}\right)
$$

Let $Q^{n-m} \subset Q^{n}$ be the intersection of $Q^{n}$ with a linear subspace of codimension $m$ containing $p$ such that $Q^{n-m}$ is smooth at $p$. Since the fiber of $\pi_{m}$ over $L^{m} \subset Q^{n}$ is $L^{m}$, we see that the restriction

$$
\pi_{m}: \pi_{0}^{-1}\left(Q^{n-m}\right) \rightarrow \mathrm{OG}\left(\mathbb{P}^{m}, Q^{n}\right)
$$

is birational. By (57 4), $\pi_{0}$ is Zariski locally trivial with fiber $\mathrm{OG}\left(\mathbb{P}^{m-1}, Q_{W}^{n-2}\right)$. Projecting from $p$ shows that $Q^{n-m} \stackrel{\text { bir }}{\sim} \mathbb{P}^{n-m}$. Thus

$$
\mathrm{OG}\left(\mathbb{P}^{m}, Q^{n}\right) \stackrel{\text { bir }}{\sim} \mathrm{OG}\left(\mathbb{P}^{m-1}, Q_{W}^{n-2}\right) \times Q^{n-m} \stackrel{\text { bir }}{\sim} \mathrm{OG}\left(\mathbb{P}^{m-1}, Q_{W}^{n-2}\right) \times \mathbb{P}^{n-m}
$$

Corollary 59. Let $Q^{n}$ be a quadric with Witt index $r$ and anisotropic reduction $Q_{\mathrm{an}}^{n-2 r}$. 
(1) If $m<r$ then $\mathrm{OG}\left(\mathbb{P}^{m}, Q^{n}\right)$ is rational.

(2) If $m \geq r$ then $\mathrm{OG}\left(\mathbb{P}^{m}, Q^{n}\right)(k)=\emptyset$ and

$$
\mathrm{OG}\left(\mathbb{P}^{m}, Q^{n}\right) \stackrel{b i r}{\sim} \mathrm{OG}\left(\mathbb{P}^{m-r}, Q_{\mathrm{an}}^{n-2 r}\right) \times \mathbb{P}^{r(2 n-r+1) / 2} .
$$

Proof. The claims are clear if the Witt index is 0. Otherwise we apply (58) repeatedly.

If $m \geq r$ we stop when we reach the anisotropic kernel $Q_{\text {an }}^{n-2 r}$ to get (2). If $m<r$ then we stop with $\mathrm{OG}\left(\mathbb{P}^{0}, Q_{W}^{n-2 m}\right)=Q_{W}^{n-2 m}$. Since $m<r$ the quadric $Q_{W}^{n-2 m}$ still has $k$-points, hence it is birational to $\mathbb{P}^{n-2 m}$. Thus $\mathrm{OG}\left(\mathbb{P}^{m}, Q^{n}\right)$ is rational.

We get the following rationality criteria for $\mathrm{OG}\left(\mathbb{P}^{m}, Q^{n}\right)$.

Theorem 60. Let $Q^{n}=\left(q\left(x_{0}, \ldots, x_{n+1}\right)=0\right)$ be a smooth quadric over a field $k$. The following are equivalent.

(1) $\mathrm{OG}\left(\mathbb{P}^{m}, Q^{n}\right)$ is rational.

(2) $\mathrm{OG}\left(\mathbb{P}^{m}, Q^{n}\right)$ has a $k$-point.

(3) $\mathrm{OG}\left(\mathbb{P}^{m}, Q^{n}\right)$ has a $k^{\prime}$-point over an odd degree field extension $k^{\prime} / k$.

(4) $Q^{n}$ contains a linear subspace of dimension $m$.

(5) The Witt index of $q$ is $\geq m+1$. Equivalently, we can write

$$
q\left(x_{0}, \ldots, x_{n+1}\right) \sim y_{0} y_{1}+\cdots+y_{2 m} y_{2 m+1}+q_{W}\left(y_{2 m+2}, \ldots, y_{n+1}\right) .
$$

Proof. The implications (1) $\Rightarrow(2) \Rightarrow(3)$ and $(2) \Leftrightarrow(4)$ are clear and (4) $\Leftrightarrow(5)$ was noted in Paragraph 20, We proved in (59) that $(5) \Rightarrow(1)$. Thus it remains to show that $(3) \Rightarrow(2)$.

We use induction on $n$. If $\mathrm{OG}\left(\mathbb{P}^{m}, Q^{n}\right)$ has a $k^{\prime}$-point then $Q^{n}$ contains a linear space defined over $k^{\prime}$, hence also a $k^{\prime}$-point. Thus there is a $k$-point $p \in Q^{n}$ by Springer's theorem; see Spr52 or (67). By (58)

$$
\mathrm{OG}\left(\mathbb{P}^{m}, Q^{n}\right) \stackrel{\text { bir }}{\sim} \mathrm{OG}\left(\mathbb{P}^{m-1}, Q_{W}^{n-2}\right) \times \mathbb{P}^{n},
$$

hence $\mathrm{OG}\left(\mathbb{P}^{m-1}, Q_{W}^{n-2}\right)$ also has a $k^{\prime}$-point. By induction $\mathrm{OG}\left(\mathbb{P}^{m-1}, Q_{W}^{n-2}\right)$ has a $k$-point, hence, using $(60,6)$ from right to left shows that $\mathrm{OG}\left(\mathbb{P}^{m}, Q^{n}\right)$ has a $k$-point.

Three of the orthogonal Grassmannians are closely related to Severi-Brauer varieties. The obvious one is $\mathrm{OG}\left(\mathbb{P}^{1}, Q^{2}\right)$, the more interesting examples are $\mathrm{OG}\left(\mathbb{P}^{1}, Q^{3}\right)$ and $\mathrm{OG}\left(\mathbb{P}^{2}, Q^{4}\right)$. For these the symmetric powers can be determined using [Kol16b]. (See Kol16a for a geometric introduction to Severi-Brauer varieties.)

Lemma 61. Let $Q^{3}=\left(q\left(x_{0}, \ldots, x_{4}\right)=0\right)$ be a smooth quadric 3-fold over $k$. Then

(1) $\mathrm{OG}\left(\mathbb{P}^{1}, Q^{3}\right)$ is a 3-dimensional Severi-Brauer variety.

(2) $\mathrm{OG}\left(\mathbb{P}^{1}, Q^{3}\right)$ has period 1 or 2 .

(3) $\mathrm{OG}\left(\mathbb{P}^{1}, Q^{3}\right) \cong \mathbb{P}^{3} \Leftrightarrow Q^{3}$ contains a line $\Leftrightarrow q \sim y_{0} y_{1}+y_{2} y_{3}+a_{4} y_{4}^{2}$.

(4) $\mathrm{OG}\left(\mathbb{P}^{1}, Q^{3}\right)$ has index 2 iff $q \sim q_{4}\left(y_{0}, \ldots, y_{3}\right)+a_{4} y_{4}^{2}$ such that $\operatorname{discr}\left(q_{4}\right)$ is a square. If these hold then $\operatorname{Sym}^{2}\left(\mathrm{OG}\left(\mathbb{P}^{1}, Q^{3}\right)\right) \stackrel{\text { bir }}{\sim} \mathbb{P}^{6}$.

(5) $\mathrm{OG}\left(\mathbb{P}^{1}, Q^{3}\right)$ has index 4 iff $\operatorname{Sym}^{2}\left(\mathrm{OG}\left(\mathbb{P}^{1}, Q^{3}\right)\right)$ has no k-points.

(6) Let $a_{0} x_{0}^{2}+\cdots+a_{4} x_{4}^{2}=0$ be a diagonal equation of $Q^{3}$. Then

$\left[\mathrm{OG}\left(\mathbb{P}^{1}, Q^{3}\right)\right]=\left[\left(a_{0} x_{0}^{2}+a_{1} x_{1}^{2}+a_{2} x_{2}^{2}=0\right)\right] \times\left[\left(a_{0} a_{1} a_{2} y^{2}=a_{3} x_{3}^{2}+a_{4} x_{4}^{2}\right)\right]$, where $[*]$ denotes the class of an object in the Brauer group. 
Proof. Assume first that $k$ is algebraically closed and let $\ell \in Q^{3}$ be any line. Then $\left\{\ell^{\prime}: \ell \cap \ell^{\prime} \neq \emptyset\right\}$ defines a divisor $H_{\ell} \subset \mathrm{OG}(2,5)$. Given three general lines $\ell_{1}, \ell_{2}, \ell_{3}$, there is a unique line $\ell^{\prime}$ that meets all 3 . Indeed, the first 2 lines span an $L^{3} \subset \mathbb{P}^{4}$ and $\ell_{3} \cap L^{3}$ is a single point $p$. Thus the only line $\ell^{\prime}$ in $\mathbb{P}^{4}$ that meets the $\ell_{i}$ is the one passing through $p$ and meeting both $\ell_{1}, \ell_{2}$. This $\ell^{\prime}$ meets $Q^{3}$ in 3 points,so is contained in it. This shows that $\left(H^{3}\right)=1$, so $|H|$ maps $\mathrm{OG}(2,5)$ isomorphically onto $\mathbb{P}^{3}$, proving (1).

An $m$-dimensional Severi-Brauer variety is isomorphic to $\mathbb{P}^{m}$ iff it has a $k$-point. Thus $\mathrm{OG}\left(\mathbb{P}^{1}, Q^{3}\right) \cong \mathbb{P}^{3}$ iff $Q^{3}$ contains a line and the rest of $(3)$ is clear.

If we are over a field $k$, then there may not be lines defined over $k$ but there are conics $C \subset Q^{3}$ defined over $k$. Thus $\left\{\ell^{\prime}: C \cap \ell^{\prime} \neq \emptyset\right\}$ defines a divisor $D_{C} \subset$ $\mathrm{OG}\left(\mathbb{P}^{1}, Q^{3}\right)$ that is defined over $k$. Over $\bar{k}$ we have $D_{C} \sim 2 H$ and $\ell^{\prime} \mapsto C \cap \ell^{\prime}$ gives a morphism $D_{C} \rightarrow C$ defined over $k$. Thus in fact $D$ is a product of 2 conics $D_{C} \cong C \times C^{\prime}$. This shows that $\mathrm{OG}\left(\mathbb{P}^{1}, Q^{3}\right)$ is a Severi-Brauer variety of period 2 , proving (2). (By our construction, it contains a quadric that is a product of 2 conics; actually this always holds, by a theorem of Albert, if the period is 2; see Kol05, Thm.18] for a geometric proof.)

By definition, OG $\left(\mathbb{P}^{1}, Q^{3}\right)$ has index 2 iff it has a degree 2 point, this $Q^{3}$ contains a conjugate pair of lines $\ell, \ell^{\prime}$. These span an $L^{3} \subset \mathbb{P}^{4}$ and $Q^{2}:=L^{3} \cap Q^{3}$ is a smooth quadric that contains a conjugate pair of lines. By (62) this holds iff its discriminant is a square.

Next we claim that if $P$ is a Severi-Brauer variety that has a point over a degree 2 field extension $k^{\prime} / k$ then $\mathrm{Sym}^{2} P$ is rational. Indeed, let $\pi: U \rightarrow G$ denote the universal bundle over the Grassmannian of lines. Then $\operatorname{Sym}^{2} P$ is birational to $\mathbb{P}_{G}\left(\pi_{*} \mathcal{O}_{U}\left(-K_{U}\right)\right)$, hence to $\mathbb{P}^{2} \times G$. Let $L$ be a hyperplane in $P$ defined over $k^{\prime}$. Then $G$ is birational to the Weil restriction $\Re_{k^{\prime} / k} L$, hence rational and so is $\mathrm{Sym}^{2} P$. This proves (4) and (5) just lists the remaining cases.

For (6) we need to follow the construction of $D_{C}$ above. So assume that $Q^{3}=$ $\left(a_{0} x_{0}+\cdots+a_{4} x_{4}^{2}=0\right)$. We can use $C=\left(x_{3}=x_{4}=0\right)$ as our first conic; it is isomorphic to $\left(a_{0} x_{0}^{2}+a_{1} x_{1}^{2}+a_{2} x_{2}^{2}=0\right) \subset \mathbb{P}^{2}$. To get our second conic $C^{\prime}$, we project $Q^{3}$ from $C$ to get $\pi: Q^{3} \rightarrow \mathbb{P}_{x_{3}, x_{4}}^{1}$. The fiber over a point $(s: t)$ is

$$
\begin{aligned}
Q^{2}(s, t) & =\left(a_{0} x_{0}^{2}+\cdots+a_{4} x_{4}^{2}=t x_{3}-s x_{4}=0\right) \\
& \cong\left(a_{0} x_{0}^{2}+a_{1} x_{1}^{2}+a_{2} x_{2}^{2}+\left(a_{3} s^{2}+a_{4} t^{2}\right) x_{3}^{2}=0\right) .
\end{aligned}
$$

Furthermore, $C^{\prime} \rightarrow \mathbb{P}_{x_{3}, x_{4}}^{1}$ is the double cover whose fiber over $(s: t)$ is the 2 families of lines on $Q^{2}(s, t)$. Thus, by (62), the equation of $C^{\prime}$ is $w^{2}=\operatorname{discr}\left(Q^{2}(s, t)\right)$. We can divide by $a_{0} a_{1} a_{2}$ and set $y:=w /\left(a_{0} a_{1} a_{2}\right)$ to get the form $C^{\prime} \cong\left(a_{0} a_{1} a_{2} y^{2}=\right.$ $\left.a_{3} x_{3}^{2}+a_{4} x_{4}^{2}\right)$.

As we noted, the middle dimensional orthogonal Grassmannians $\mathrm{OG}\left(\mathbb{P}^{r}, Q^{2 r}\right)$ are geometrically reducible. The discriminant tells us when they are reducible.

Lemma 62. Let $Q^{2 r}$ be an even dimensional smooth quadric over a field $k$. Then the scheme parametrizing the 2 geometric components of $\mathrm{OG}\left(\mathbb{P}^{r}, Q^{2 r}\right)$ is $\left(t^{2}+\right.$ $\left.(-1)^{r} \Delta=0\right)$, where $\Delta$ is the discriminant of $Q^{2 r}$.

Thus $\mathrm{OG}\left(\mathbb{P}^{r}, Q^{2 r}\right)$ is reducible iff $(-1)^{r+1} \Delta\left(Q^{2 r}\right)$ is a square.

Proof. Set $m:=r+1$ and consider the universal quadratic form

$$
q_{\text {univ }}^{2 m}\left(x_{1}, \ldots, x_{2 m}\right):=\sum_{1 \leq i \leq j \leq 2 m} t_{i j} x_{i} x_{j}
$$


over the affine space of dimension $m(2 m+1)$ over Spec $\mathbb{Z}\left[\frac{1}{2}\right]$. Let $\Delta$ be the discriminant and $U:=\mathbb{A}^{m(2 m+1)} \backslash(\Delta=0)$ the complement of its zero set. The Stein factorization of the universal orthogonal Grassmannian of $m$-dimensional isotropic subspaces gives an étale double cover of $U$. Since $\mathbb{A}^{m(2 m+1)}$ is simply connected, this is given by an equation

$$
z^{2}=(-1)^{a} \cdot 2^{b} \cdot \Delta^{c}
$$

for some integers $a, b, c$ which can all be chosen 0 or 1 . To pin down these choices, we can check a particular case, for instance

$$
q_{t}:=x_{1}^{2}-x_{2}^{2}+\cdots+x_{2 m-3}^{2}-x_{2 m-2}^{2}+x_{2 m-1}^{2}-t x_{2 m}^{2} .
$$

We have $\Delta\left(q_{t}\right)=(-1)^{m} t$ and the 2 families of $m$-planes correspond to the members

$$
\left(x_{1}-x_{2}=\cdots=x_{2 n-3}-x_{2 n-2}=x_{2 m-1} \pm \sqrt{t} x_{2 m}=0 .\right.
$$

Thus the correct equation is $z^{2}=(-1)^{m} \Delta$.

Proposition 63. Let $Q^{2 r}$ be an even dimensional smooth quadric over a field $k$ and $Q^{2 r-1} \subset Q^{2 r}$ a smooth quadric hypersurface. Them

$$
\mathrm{OG}\left(\mathbb{P}^{r}, Q^{2 r}\right) \cong \mathrm{OG}\left(\mathbb{P}^{r-1}, Q^{2 r-1}\right) \times\left(t^{2}+(-1)^{r} \Delta=0\right) .
$$

Proof. Intersecting $L^{r} \subset Q^{2 r}$ with $Q^{2 r-1}$ gives a morphism $\pi_{1}: \mathrm{OG}\left(\mathbb{P}^{r}, Q^{2 r}\right) \rightarrow$ $\mathrm{OG}\left(\mathbb{P}^{r-1}, Q^{2 r-1}\right)$ which is everywhere defined since a smooth quadric of dimension $2 r-1$ can not contain a $\mathbb{P}^{r}$. We can think of a fiber of $\pi_{1}$ as a fiber of the projection considered in $(57,3)$

$$
\mathrm{OG}\left(\mathbb{P}^{r-1}, Q^{2 r}\right) \stackrel{\pi_{r-1}}{\longleftarrow} \mathrm{OF}\left(\mathbb{P}^{r-1}, \mathbb{P}^{r}, Q^{2 r}\right) .
$$

We identified this fiber as a 0 -dimensional quadric, hence 2 points. Combining with (62) we get a surjection

$$
\mathrm{OG}\left(\mathbb{P}^{r}, Q^{2 r}\right) \rightarrow \mathrm{OG}\left(\mathbb{P}^{r-1}, Q^{2 r-1}\right) \times\left(t^{2}+(-1)^{r} \Delta=0\right),
$$

which is then necessarily an isomorphism.

Corollary 64. $\mathrm{OG}\left(\mathbb{P}^{2}, Q^{4}\right)$ is a Severi-Brauer scheme over $\left(t^{2}+\Delta=0\right)$.

\section{Symmetric squares of orthogonal Grassmannians}

In this section we study the rationality of $\operatorname{Sym}^{2}\left(\mathrm{OG}\left(\mathbb{P}^{1}, Q^{n}\right)\right)$. Note that if $n \geq 3$ then $\operatorname{Aut}\left(Q^{n}\right)$ acts with a dense orbit on $\operatorname{Sym}^{2}\left(\mathrm{OG}\left(\mathbb{P}^{1}, Q^{n}\right)\right)$. Thus if the latter has a $k$-point then it is unirational. (This needs a small argument about singular $k$-points.)

More generally, one can ask about the structure of $\operatorname{Sym}^{d}\left(\mathrm{OG}\left(\mathbb{P}^{m}, Q^{n}\right)\right)$. Building on the assumption that quadrics are similar to Severi-Brauer varieties, the following questions appear to be quite natural and they should give a general outline of what could hold. The analogous assertions for Severi-Brauer varieties are proved in Kol16b, however, similar problems about other homogeneous spaces turned out to have a negative answer [Flo04, Par05]. So there is little reason to believe that the answers should be positive.

Question 65. Let $Q^{n}$ be a smooth quadric over a field $k$. Fix $m \geq 0$ and write $d=2^{a} d^{\prime}$ where $d^{\prime}$ is odd. Which of the following are true?

(1) $\operatorname{Sym}^{d}\left(\mathrm{OG}\left(\mathbb{P}^{m}, Q^{n}\right)\right)$ is rational over $k$ if $2^{m+1} \mid d$. 
(2) $\operatorname{Sym}^{d}\left(\mathrm{OG}\left(\mathbb{P}^{m}, Q^{n}\right)\right)$ is rational over $k$ iff it has a $k$-point.

(3) The stable birational equivalence class of $\operatorname{Sym}^{d}\left(\mathrm{OG}\left(\mathbb{P}^{m}, Q^{n}\right)\right)$ depends only on $2^{a}$.

The simplest case, when $a=0$, follows from (60).

Proposition 66. Let $Q^{n}$ be a smooth quadric over a field $k$ and assume that $d$ is odd. Then

$$
\operatorname{Sym}^{d}\left(\mathrm{OG}\left(\mathbb{P}^{m}, Q^{n}\right)\right) \stackrel{\text { stab }}{\sim} \mathrm{OG}\left(\mathbb{P}^{m}, Q^{n}\right) .
$$

Proof. We show the stable birational equivalences

$$
\operatorname{Sym}^{d}\left(\mathrm{OG}\left(\mathbb{P}^{m}, Q^{n}\right)\right) \stackrel{\text { stab }}{\sim} \operatorname{Sym}^{d}\left(\mathrm{OG}\left(\mathbb{P}^{m}, Q^{n}\right)\right) \times \mathrm{OG}\left(\mathbb{P}^{m}, Q^{n}\right) \stackrel{\text { stab }}{\sim} \mathrm{OG}\left(\mathbb{P}^{m}, Q^{n}\right) .
$$

First let $K$ be the function field of $\mathrm{OG}\left(\mathbb{P}^{m}, Q^{n}\right)$. Then $\mathrm{OG}\left(\mathbb{P}^{m}, Q^{n}\right)(K) \neq \emptyset$ hence $\operatorname{OG}\left(\mathbb{P}^{m}, Q^{n}\right)$ is rational over $K$ by $(60)$ and so are its symmetric powers. This shows the stable birational equivalence on the right.

Next let $L$ be the function field of $\operatorname{Sym}^{d}\left(\mathrm{OG}\left(\mathbb{P}^{m}, Q^{n}\right)\right)$. Then $\mathrm{OG}\left(\mathbb{P}^{m}, Q^{n}\right)$ has a point in an odd degree field extension $L^{\prime} / L$, hence it is rational over $L$ by (60). Thus the stable birational equivalence on the left also holds.

Another special case of Question 65 is symmetric powers of $\mathrm{OG}\left(\mathbb{P}^{0}, Q^{n}\right) \cong Q^{n}$. Spr52 proves that if a quadric has a point over an odd degree field extension of $k$ then it has a $k$-point. The idea of the proof gives more.

Theorem 67. Let $Q^{n}$ be a smooth quadric of dimension $n \geq 1$. Then

$$
\operatorname{Sym}^{d}\left(Q^{n}\right) \stackrel{\text { bir }}{\sim} \begin{cases}\mathbb{P}^{n d} & \text { if } d \text { is even, } \\ Q^{n} \times \mathbb{P}^{n d-n} & \text { if d is odd. }\end{cases}
$$

Proof. We prove that, for $d \geq 2, \operatorname{Sym}^{d}\left(Q^{n}\right) \stackrel{\text { bir }}{\sim} \operatorname{Sym}^{d-2}\left(Q^{n}\right) \times \mathbb{P}^{2 n}$. In affine coordinates we write $Q^{n}=\left(q\left(x_{1}, \ldots, x_{n}, t\right)=0\right)$.

For $d$ general points $p_{1}, \ldots, p_{d} \in Q^{n}$ there are unique polynomials $g_{1}(t), \ldots, g_{n}(t)$ of degree $\leq d-1$ whose graph $\Gamma:=\Gamma\left(p_{1}, \ldots, p_{d}\right)$ passes through $p_{1}, \ldots, p_{d}$. We define $\operatorname{Sym}^{d}\left(Q^{n}\right) \rightarrow \operatorname{Sym}^{d-2}\left(Q^{n}\right) \times \mathbb{A}^{n} \times \mathbb{A}^{n}$ by sending $\left\{p_{1}, \ldots, p_{d}\right\}$ to the triple consisting of the $d-2$ other intersection points of $\Gamma$ with $Q^{n}$ and the intersection points of $\Gamma$ with the hyperplanes $(t=0)$ and $(t=1)$.

68 (Witt reduction and symmetric powers). The rationality problem of symmetric powers can be reduced to the anisotropic case. Indeed, we proved in (58) that if $Q^{n}$ is a smooth quadric such that $Q^{n}(k) \neq \emptyset$ then

$$
\mathrm{OG}\left(\mathbb{P}^{m}, Q^{n}\right) \stackrel{\text { bir }}{\sim} \mathrm{OG}\left(\mathbb{P}^{m-1}, Q_{W}^{n-2}\right) \times \mathbb{P}^{n-m} .
$$

We can next take symmetric powers and, using [Kol16b, Lem.7], conclude that

$$
\operatorname{Sym}^{d}\left(\mathrm{OG}\left(\mathbb{P}^{m}, Q^{n}\right)\right) \stackrel{\text { bir }}{\sim} \operatorname{Sym}^{d}\left(\mathrm{OG}\left(\mathbb{P}^{m-1}, Q_{W}^{n-2}\right)\right) \times \mathbb{P}^{d(n-m)} .
$$

69 (Symmetric square of $\left.\mathrm{OG}\left(\mathbb{P}^{r}, Q^{2 r}\right)\right)$. Since $\mathrm{OG}\left(\mathbb{P}^{r}, Q^{2 r}\right)$ has 2 geometric irreducible components, its symmetric square can be written as

$$
\operatorname{Sym}^{2}\left(\mathrm{OG}\left(\mathbb{P}^{r}, Q^{2 r}\right)\right)=\operatorname{Sym}_{s}^{2}\left(\mathrm{OG}\left(\mathbb{P}^{r}, Q^{2 r}\right)\right) \amalg \operatorname{Sym}_{a}^{2}\left(\mathrm{OG}\left(\mathbb{P}^{r}, Q^{2 r}\right)\right)
$$

where the first (symmetric) component parametrizes pairs $L_{1}^{r}, L_{2}^{r} \subset Q^{2 r}$ that are in different geometric irreducible components of $\mathrm{OG}\left(\mathbb{P}^{r}, Q^{2 r}\right)$ while the second (anti-symmetric) component parametrizes pairs that are in the same geometric 
irreducible component. Note that $\operatorname{Sym}_{s}^{2}\left(\mathrm{OG}\left(\mathbb{P}^{r}, Q^{2 r}\right)\right)$ is geometrically irreducible while $\operatorname{Sym}_{a}^{2}\left(\mathrm{OG}\left(\mathbb{P}^{r}, Q^{2 r}\right)\right)$ is geometrically reducible.

If $r=1$ then

$$
\begin{aligned}
& \operatorname{Sym}_{s}^{2}\left(\mathrm{OG}\left(\mathbb{P}^{1}, Q^{2}\right)\right) \cong Q^{2} \text { and } \\
& \operatorname{Sym}_{a}^{2}\left(\mathrm{OG}\left(\mathbb{P}^{1}, Q^{2}\right)\right) \cong\left(t^{2}=\Delta\right) \times \mathbb{P}^{2}
\end{aligned}
$$

Indeed, the first isomorphism is obtained by identifying a pair of intersecting lines on $Q^{2}$ with their intersection point. The second isomorphism is obtained by noting that $\mathrm{OG}\left(\mathbb{P}^{1}, Q^{2}\right)$ is a conic bundle over $\left(t^{2}=\Delta\right)$ by (62) and the symmetric square of any conic is $\mathbb{P}^{2}$ by (67).

If $r=2$ then two general planes of the same family meet at a single point, thus we have a map

$$
\operatorname{Sym}_{a}^{2}\left(\mathrm{OG}\left(\mathbb{P}^{2}, Q^{4}\right)\right) \rightarrow Q^{4} .
$$

The fiber over $p \in Q^{4}$ can be identified with $\operatorname{Sym}_{a}^{2}\left(\mathrm{OG}\left(\mathbb{P}^{1}, Q_{p}^{2}\right)\right)$. The $\mathrm{OG}\left(\mathbb{P}^{1}, Q_{p}^{2}\right)$ bundle over $Q^{4}$ is a conic bundle over an étale double cover of $Q^{4}$. Since $Q^{4}$ is simply connected, the étale double cover is isomorphic to $Q^{4} \times\left(t^{2}+\Delta=0\right)$. (Note that Witt reduction from $Q^{4}$ to $Q_{p}^{2}$ changes the sign of the discriminant.) Thus we have a conic bundle over $Q^{4} \times\left(t^{2}+\Delta=0\right)$ and we take its relative symmetric square. This is a Zariski locally trivial $\mathbb{P}^{2}$-bundle over $Q^{4} \times\left(t^{2}+\Delta=0\right)$, hence

$$
\operatorname{Sym}_{a}^{2}\left(\mathrm{OG}\left(\mathbb{P}^{2}, Q^{4}\right)\right) \stackrel{\text { bir }}{\sim}\left(t^{2}+\Delta=0\right) \times Q^{4} \times \mathbb{P}^{2} .
$$

Symmetric squares of $\mathrm{OG}\left(\mathbb{P}^{1}, Q^{n}\right)$ are related to double covers of Grassmannians.

Proposition 70. Let $Q^{n}$ be a smooth quadric of dimension $\geq 3$. Then there is a double cover $\pi: G\left(Q^{n}\right) \rightarrow \operatorname{Grass}\left(\mathbb{P}^{3}, \mathbb{P}^{n+1}\right)$, ramified along a divisor $D \sim 2 H$ such that

$$
\operatorname{Sym}^{2}\left(\mathrm{OG}\left(\mathbb{P}^{1}, Q^{n}\right)\right) \stackrel{\text { bir }}{\sim} G\left(Q^{n}\right) \times \mathbb{P}^{2} .
$$

Proof. A $K$-point of $\operatorname{Sym}^{2}\left(\mathrm{OG}\left(\mathbb{P}^{1}, Q^{n}\right)\right)$ is the same as a pair of lines $\ell, \ell^{\prime} \subset Q^{n}$ that are conjugate over $K$. In general $\ell, \ell^{\prime}$ are disjoint, so their span $\left\langle\ell, \ell^{\prime}\right\rangle$ has dimension 3. Thus we get a map

$$
\pi: \operatorname{Sym}^{2}\left(\mathrm{OG}\left(\mathbb{P}^{1}, Q^{3}\right)\right) \rightarrow \operatorname{Grass}\left(\mathbb{P}^{3}, \mathbb{P}^{n+1}\right) .
$$

Given $L^{3} \subset \mathbb{P}^{n+1}$ such that $Q^{n} \cap L^{3}$ is a smooth quadric surface $Q_{L}^{2}$, we proved in [69, 2) that the fiber of $\pi$ over $\left[L^{3}\right]$ is

$$
\operatorname{Sym}_{a}^{2}\left(\mathrm{OG}\left(\mathbb{P}^{1}, Q^{2}\right)\right) \cong\left(t^{2}=\Delta\right) \times \mathbb{P}^{2} .
$$

Therefore the Stein factorization of $\pi$ is

$$
\operatorname{Sym}^{2}\left(\mathrm{OG}\left(\mathbb{P}^{1}, Q^{3}\right) \stackrel{\text { bir }}{\sim} G\left(Q^{n}\right) \times \mathbb{P}^{2} \rightarrow G\left(Q^{n}\right) \rightarrow \operatorname{Grass}\left(\mathbb{P}^{3}, \mathbb{P}^{n+1}\right),\right.
$$

where $G\left(Q^{n}\right) \rightarrow \operatorname{Grass}\left(\mathbb{P}^{3}, \mathbb{P}^{n+1}\right)$ is a double cover that ramifies over a point of the Grassmannian $\left[L^{3}\right] \in \operatorname{Grass}\left(\mathbb{P}^{3}, \mathbb{P}^{n+1}\right)$ iff $Q^{n} \cap L^{3}$ is a singular quadric (or if $\left.L^{3} \subset Q^{n}\right)$.

Let $Q^{3} \subset \mathbb{P}^{4}$ be a smooth quadric 3-fold. A general pencil of hyperplane sections $\left\langle L_{\lambda}^{3} \cap Q^{3}\right\rangle$ of $Q^{3}$ has 2 singular members. Since such pencils $\left\langle L_{\lambda}^{3}\right\rangle \subset L^{4} \subset \mathbb{P}^{n+1}$ correspond to lines on $\operatorname{Grass}\left(\mathbb{P}^{3}, \mathbb{P}^{n+1}\right)$, we conclude that the branch divisor $D \subset$ $\operatorname{Grass}\left(\mathbb{P}^{3}, \mathbb{P}^{n+1}\right)$ is linearly equivalent to $2 H$ where $H$ is the hyperplane class on $\operatorname{Grass}\left(\mathbb{P}^{3}, \mathbb{P}^{n+1}\right)$.

Next we show that $G\left(Q^{3}\right)$ is itself a quadric and determine its equation. This will prove Proposition 15 . 
71 (Proof of Proposition 15). By Theorem 13 and by Proposition 70 .

$$
\operatorname{Map}_{2}\left(\mathbb{P}^{2}, Q^{5}\right) \stackrel{\text { bir }}{\sim} \operatorname{Sym}^{2}\left(\mathrm{OG}\left(\mathbb{P}^{1}, Q_{W}^{3}\right)\right) \times \mathbb{P}^{20} \stackrel{\text { bir }}{\sim} G\left(Q_{W}^{3}\right) \times \mathbb{P}^{22},
$$

where $Q_{W}^{3}$ is the Witt reduction of $Q^{5}$. Thus we need to understand $\pi: G\left(Q_{W}^{3}\right) \rightarrow$ $\operatorname{Grass}\left(\mathbb{P}^{3}, \mathbb{P}^{4}\right)$. Note that Grass $\left(\mathbb{P}^{3}, \mathbb{P}^{4}\right)$ is the dual of $\mathbb{P}^{4}$ and $\pi$ ramifies along the dual quadric $Q_{W}^{\vee}$ which is isomorphic to $Q_{W}^{3}$. Thus if $Q_{W}^{3} \cong\left(q\left(x_{0}, \ldots, x_{4}\right)=0\right)$ then

$$
G\left(Q_{W}^{3}\right) \cong\left(c \Delta(q) z^{2}=q\left(x_{0}, \ldots, x_{4}\right)\right) \text { for some } c .
$$

It is not hard to compute everything explicitly and obtain that $c=1$. As a possible shortcut, working with the universal quadric over $\mathbb{Z}\left[\frac{1}{2}\right]$ as in the proof of (62), we see that $c$ is a unit in $\mathbb{Z}\left[\frac{1}{2}\right]$, hence it is enough to compute it in one example.

Acknowledgments. I thank I. Coskun, G. Di Cerbo, D. Krashen, R. Kusner, M. Lieblich, N. Lubbes, R. Parimala, B. Poonen, M. Skopenkov and B. Sturmfels for comments, discussions and references. I learned a lot of the early history, especially the role of [Ber1907], from F. Russo. Partial financial support was provided by the NSF under grant number DMS-1362960.

\section{REFERENCES}

[AK03] Carolina Araujo and János Kollár, Rational curves on varieties, Higher dimensional varieties and rational points (Budapest, 2001), Bolyai Soc. Math. Stud., vol. 12, Springer, Berlin, 2003, pp. 13-68.

[Ber1907] Eugenio Bertini, Introduzione alla geometria proiettiva degli iperspazi con appendice sulle curve algebriche e loro singolaritá, E. Spoerri, Pisa, 1907.

[Ber24] _ Einfü̈hrung in die projektive Geometrie mehrdimensionaler Raäume, mit einem Anhang über algebraische Kurven und ihre Singularitäten, Seidel \& Sohn, Wien, 1924.

[BKT15] Anders Skovsted Buch, Andrew Kresch, and Harry Tamvakis, A Giambelli formula for even orthogonal Grassmannians, J. Reine Angew. Math. 708 (2015), 17-48. MR 3420327

[Cas1894] Guido Castelnuovo, Sulle superficie algebriche che ammettono un sistema doppiamente infinito di sezioni piane riduttibili, Rendiconti della E. Acc. dei Lincei 5 (1894), 22-25.

[Cob22] Arthur B. Coble, Associated sets of points, Trans. Amer. Math. Soc. 24 (1922), no. 1, 1-20. MR 1501210

[Cos14] Izzet Coskun, Rigidity of Schubert classes in orthogonal Grassmannians, Israel J. Math. 200 (2014), no. 1, 85-126. MR 3219572

[Dal85] Magnar Dale, Severi's theorem on the Veronese-surface, J. London Math. Soc. (2) 32 (1985), no. 3, 419-425. MR 825917

[ESB89] Lawrence Ein and Nicholas Shepherd-Barron, Some special Cremona transformations, Amer. J. Math. 111 (1989), no. 5, 783-800. MR 1020829

[Flo04] Mathieu Florence, Zéro-cycles de degré un sur les espaces homogènes, Int. Math. Res. Not. (2004), no. 54, 2897-2914. MR 2097288

[FP97] W. Fulton and R. Pandharipande, Notes on stable maps and quantum cohomology, Algebraic geometry - Santa Cruz 1995, Proc. Sympos. Pure Math., vol. 62, Amer. Math. Soc., Providence, RI, 1997, pp. 45-96.

[HP47] W. V. D. Hodge and D. Pedoe, Methods of Algebraic Geometry. Vols. I-III., Cambridge, at the University Press, 1947. MR 0028055 (10,396b)

[HRS04] Joe Harris, Mike Roth, and Jason Starr, Rational curves on hypersurfaces of low degree, J. Reine Angew. Math. 571 (2004), 73-106. MR 2070144 (2005e:14067)

[IR10] Paltin Ionescu and Francesco Russo, Conic-connected manifolds, J. Reine Angew. Math. 644 (2010), 145-157. MR 2671777

[Kac99] Yasuyuki Kachi, Characterization of Veronese varieties and the Hodge index theorem, (preprint), 1999. 
[Kol96] János Kollár, Rational curves on algebraic varieties, Ergebnisse der Mathematik und ihrer Grenzgebiete. 3. Folge., vol. 32, Springer-Verlag, Berlin, 1996.

[Kol05] _ Conics in the Grothendieck ring, Adv. Math. 198 (2005), no. 1, 27-35. MR 2183248 (2006k:14064)

[Kol08] L Looking for rational curves on cubic hypersurfaces, Higher-dimensional geometry over finite fields, NATO Sci. Peace Secur. Ser. D Inf. Commun. Secur., vol. 16, IOS, Amsterdam, 2008, Notes by Ulrich Derenthal, pp. 92-122. MR 2484078 (2009k:14047)

[Kol10] Holomorphic and pseudo-holomorphic curves on rationally connected varieties, Port. Math. 67 (2010), no. 2, 155-179. MR 2662865

[Kol16a] _ Severi-Brauer varieties; a geometric treatment, ArXiv e-prints (2016).

[Kol16b] — Symmetric powers of Severi-Brauer varieties, ArXiv e-prints (2016).

[Kov94] Sándor J Kovács, The cone of curves of a K3 surface, Math. Ann. 300 (1994), no. 4, 681-691. MR MR1314742 (96a:14044)

[KP77] Nicolaas H. Kuiper and William F. Pohl, Tight topological embeddings of the real projective plane in $E^{5}$, Invent. Math. 42 (1977), 177-199. MR 0494122

[KP01] B. Kim and R. Pandharipande, The connectedness of the moduli space of maps to homogeneous spaces, Symplectic geometry and mirror symmetry (Seoul, 2000), World Sci. Publ., River Edge, NJ, 2001, pp. 187-201. MR 1882330 (2002k:14021)

[KT04] Andrew Kresch and Harry Tamvakis, Quantum cohomology of orthogonal Grassmannians, Compos. Math. 140 (2004), no. 2, 482-500. MR 2027200 (2005d:14080)

[Kui62] Nicolaas H. Kuiper, On convex maps, Nieuw Arch. Wisk. (3) 10 (1962), 147-164. MR 0145545

[LP71] John A. Little and William F. Pohl, On tight immersions of maximal codimension, Invent. Math. 13 (1971), 179-204. MR 0293645

[Mel99] Massimiliano Mella, Existence of good divisors on Mukai varieties, J. Algebraic Geom. 8 (1999), no. 2, 197-206. MR MR1675146 (2000b:14049)

[Muk89] Shigeru Mukai, Biregular classification of Fano 3-folds and Fano manifolds of coindex 3, Proc. Nat. Acad. Sci. U.S.A. 86 (1989), no. 9, 3000-3002. MR 995400

[Par05] R. Parimala, Homogeneous varieties - zero-cycles of degree one versus rational points, Asian J. Math. 9 (2005), no. 2, 251-256. MR 2176607

[PR14] Luc Pirio and Francesco Russo, Quadro-quadric Cremona transformations in low dimensions via the JC-correspondence, Ann. Inst. Fourier (Grenoble) 64 (2014), no. 1, 71-111. MR 3330541

[PSS12] Helmut Pottmann, Ling Shi, and Mikhail Skopenkov, Darboux cyclides and webs from circles, Comput. Aided Geom. Design 29 (2012), no. 1, 77-97. MR 2871363

[Rus16] Francesco Russo, On the geometry of some special projective varieties, Lecture Notes of the Unione Matematica Italiana, vol. 18, Springer, Cham; Unione Matematica Italiana, Bologna, 2016. MR 3445582

[Sch01] Josef Schicho, The multiple conical surfaces, Beiträge Algebra Geom. 42 (2001), no. 1, 71-87. MR 1824751 (2002c:14059)

[Ser73] J.-P. Serre, A course in arithmetic, Springer-Verlag, New York-Heidelberg, 1973, Translated from the French, Graduate Texts in Mathematics, No. 7. MR 0344216 (49 \#8956)

[Sev1901] Francesco Severi, Intorno ai punti doppi impropri di una superficie generale dello spazio a quattro dimensioni e ai suoi punti tripli apparenti, Rend. Circ. Mat. Palermo 15 (1901), 33-51.

[SK15] M. Skopenkov and R. Krasauskas, Surfaces containing two circles through each point and Pythagorean 6-tuples, ArXiv e-prints (2015).

[Sko15] M. Skopenkov, Surfaces containing two circles through each point, ArXiv e-prints (2015).

[Spr52] Tonny Albert Springer, Sur les formes quadratiques d'indice zéro, C. R. Acad. Sci. Paris 234 (1952), 1517-1519. MR 0047021 (13,815j)

[SR49] J. G. Semple and L. Roth, Introduction to Algebraic Geometry, Oxford, at the Clarendon Press, 1949. MR 0034048 (11,535d)

[Ver1884] Giuseppe Veronese, La superficie omaloide normale a due dimensioni e del quarto ordine dello spazio a cinque dimensioni e le sue projezioni nel piano e nello spazio ordinario, Memorie della E. Acc. dei Lincei 19 (1884), 344-371.

Princeton University, Princeton NJ 08544-1000 
kollar@math.princeton.edu 